\title{
Synthesis of Gemcitabine-( $\mathrm{C}_{4}$-amide $)$-[anti-HER2/neu] Utilizing a UV-Photoactivated Gemcitabine Intermediate: Cytotoxic Anti-Neoplastic Activity against Chemotherapeutic-Resistant Mammary Adenocarcinoma SKBr-3
}

\author{
Cody P. Coyne ${ }^{1}$, Toni Jones ${ }^{1}$, Ryan Bear ${ }^{2}$ \\ ${ }^{1}$ Department of Basic Sciences, College of Veterinary Medicine, Mississippi State University, Oktibbeha County, USA; ${ }^{2}$ Wise Cen- \\ ter, Mississippi State University, Oktibbeha County, USA. \\ Email: coyne@cvm.msstate.edu
}

Received August $10^{\text {th }}, 2012$; revised September 12 $2^{\text {th }}, 2012$; accepted September $24^{\text {th }}, 2012$

\begin{abstract}
Gemcitabine is a pyrimidine nucleoside analog that becomes triphosphorylated intracellularly where it competitively inhibits cytidine incorporation into DNA strands. Another mechanism-of-action of gemcitabine (diphosphorylated form) involves irreversible inhibition of the enzyme ribonucleotide reductase thereby preventing deoxyribonucleotide synthesis. Functioning as a potent chemotherapeutic gemcitabine promote decreases in neoplastic cell proliferation and apoptosis which is frequently found to be effective for the treatment of several leukemias and a wide spectrum of carcinomas. A brief plasma half-life in part due to rapid deamination and chemotherapeutic-resistance restricts the utility of gemcitabine in clinical oncology. Selective "targeted" delivery of gemcitabine represents a potential molecular strategy for simultaneously prolonging its plasma half-life and minimizing innocient tissues and organ systems exposure to chemotherapy. The molecular design and an organic chemistry based synthesis reaction is described that initially generates a UV-photoactivated gemcitabine intermediate. In a subsequent phase of the synthesis method the UV-photoactivated gemcitabine intermediate is covalently bonded to a monoclonal immunoglobulin yielding an end-product in the form of gemcitabine-( $\mathrm{C}_{4}$-amide)-[anti-HER2/neu]. Analysis by SDS-PAGE/chemiluminescent auto-radiography did not detect evidence of gemcitabine-( $\mathrm{C}_{4}$-amide)-[anti-HER2/neu] polymerization or degradative fragmentation while cell-ELISA demonstrated retained binding-avidity for HER2/neu trophic membrane receptor complexes highly over-expressed by chemotherapeutic-resistant mammary adenocarcinoma (SKBr-3). Compared to chemotherapeutic-resistant mammary adenocarcinoma (SKBr-3), the covalent immunochemotherapeutic, gemcitabine-( $\mathrm{C}_{4}$-amide)-[anti-HER2/neu] is anticipated to exert greater levels of cytotoxic anti-neoplastic potency against other neoplastic cell types like pancreatic carcinoma, small-cell lung carcinoma, neuroblastoma, glioblastoma, oral squamous cell carcinoma, cervical epitheliod carcinoma, or leukemia/lymphoid neoplastic cell types based on their reported sensitivity to gemcitabine and gemcitabine covalent conjugates.
\end{abstract}

Keywords: Gemcitabine; HER2/neu; UV-Photoactivated Gemcitabine-( $\mathrm{C}_{4}$-amide) Intermediate; Organic Chemistry Reaction; Gemcitabine-( $\mathrm{C}_{4}$-amide)-[anti-HER2/neu]; Covalent Bond Synthesis; Gemcitabine $\left(\mathrm{C}_{5}\right.$-methylcarbamate)-[anti-HER2/neu]; Cytotoxic Anti-Neoplastic Potency; Chemotherapeutic-Resistant; Mammary Adenocarcinoma; Cell-ELISA; SDS-PAGE; Immunodetection; Chemiluminescent Autoradiography

\section{Introduction}

The anthracyclines have historically been the most common class of chemotherapeutic covalently bonded to (large) molecular platforms that can facilitate "selective" targeted delivery [1-25]. The spectrum of anthracylines utilized to synthesize covalent anthracycline-immuno- chemotherapeutics to date has largely included doxorubicin [26-30] and to a lesser extent daunorubicin [31-33] or epirubicin $[7,34,35]$.

The chemotherapeutic, gemcitabine has in contrast to the anthracyclines been less frequently bonded covalently to large molecular weight platforms that can fa- 
cilitate selective "targeted" delivery [36]. Gemcitabine is a deoxycytidine nucleotide analog that intracellularly has a chemotherapeutic mechanism-of-action that involveds it being triphosphoralated in a manner that allows it to substitute for cytidine during DNA transcription resulting in incorporation into DNA strands and inhibition of DNA polymerase biochemical activity. A second mechanismof-action for gemcitabine involves inhibition and inactivation of ribonucleotide reductase ultimately resulting in suppression of deoxyribonucleotide synthesis in concert with diminished DNA repair and reduced DNA transcription. Each of these mechanisms-of-action collectively promotes cellular apoptosis. In clinical oncology, gemcitabine is administered for the treatment certain leukemias and potentially lymphoma conditions in addition to a spectrum of adenocarcinomas and carcinomas affecting the lung (e.g. non-small cell), pancrease, bladder and esophogus. Gemcitabine has a brief plasma half-life because it is rapidly deaminated to an inactive metabolite that is rapidly eliminated through renal excretion into the urine [37-39]. The molecular design and synthesis of a covalent gemcitabine immunochemotherapeutics provides several attributes that complement their ability to facilitate selective "targeted" delivery, progressive intracellular deposition, and more prolonged plasma pharmacokinetics for the gemcitabine moiety. Attributes in this regard presumably include steric hinderance phenomenon that accounts for gemcitabine being apparently a much poorer substrate for MDR-1 (multidrug resistance efflux pump) [40] in addition to the rapid deaminating enzyme systems, cytidine deaminase, and deoxycytidylate deaminase (following gemcitabine phosphorylation) when this chemotherapeutic is covalently incorporated into an immunochemotherapeutic.

The molecular design, synthetic organic chemistry reaction schemes, and cytotoxic anti-neoplastic potency of gemcitabine covalently bonded to large molecular weight delivery platforms has been described on only a limited scale in published reports. Due to the type and relatively low number of chemical groups (sites) available within the structure of gemcitabine there are only a small number of organic chemistry reaction schemes that have been utilized to covalently bond gemcitabine to large molecular weight platforms and very few reports have described the synthesis and cytotoxic anti-neoplastic potency of covalent gemcitabine immunochemotherapeutics [36]. The covalent bonding of gemcitabine to immunoglobulin or ligands that have binding-avidity for trophic receptors like HER2/neu and EGFR frequently over-expressed in breast cancer and by many other carcinomas or adenocarcinomas provides an opportunity to achieve additive or synergistic levels of cytotoxic anti-neoplastic potency. Monoclonal anti-HER2/neu and anti-EGFR im- munoglobulin fractions provide a molecular mechanism for achieving both selective "targeted" chemotherapeutic delivery and growth suppression of neoplastic cell types that biologically are heavily dependent on the over-expression of HER2/neu and EGFR when they function as trophic receptor complexes. Unfortunately when applied as a monotherapy, anti-HER2/neu, anti-EGFR and other therapeutic monoclonal immunoglobulin fractions reportedly have an inability to exert levels of cytotoxic activity sufficient to independently resolve many neoplastic disease states [41-47] unless they are applied in concert with conventional chemotherapy or other anticancer modalities [48,49]. Despite general familiarity with how anti-HER2/neu affects the vitality of cancer cell populations and it's application in clinical oncology, there has been surprisingly little research devoted to the molecular design, chemical synthesis and potency evaluation of covalent gemcitabine immunochemotherapetuics [36]. Even fewer reports exist to date that describe similar aspects for covalent gemcitabine-[anti-HER2/neu] immunochemotherapeutics and their potential to exert selectively "targeted" cytotoxic anti-neoplastic potency against chemotherapeutic-resis-tant mammary adenocarcinoma [36] or other cancer cell types.

\section{Materials and Methods}

\subsection{Gemcitabine-( $\mathrm{C}_{4}$-amide)-[anti-HER2/neu] Immunochemotherapeutic Synthesis}

Phase-I Synthesis Scheme for UV-Photoactivated Gemcitabine-( $\mathrm{C}_{4}$-amide $)$ Intermediates-The cytosine-like $\mathrm{C}_{4}$ amine of gemcitabine $\left(0.738 \mathrm{mg}, 2.80 \times 10^{-3}\right.$ mmoles $)$ was reacted at a 2.5:1 molar-ratio with the amine-reactive $\mathrm{N}$-hydroxysuccinimide ester "leaving" complex of succinimidyl 4,4-azipentanoate $\left(0.252 \mathrm{mg}, 1.12 \times 10^{-3}\right.$ mmoles) in the presence of triethylamine (TEA $50 \mathrm{mM}$ final concentration) utilizing dimethylsulfoxide as an anhydrous organic solvent system (Figures 1 and 2). The reaction mixture formulated from stock solutions of gemcitabine and succinimidyl 4,4-azipentanoate was continually stirred gently at $25^{\circ} \mathrm{C}$ over a 4-hour incubation period in the dark and protected from exposure to light. The relatively long incubation period of 4 hours was utilized to maximize degradation of the ester group of any residual succinimidyl 4,4-azipentanoate that may not of reacted in the first 30 to 60 minutes with the $\mathrm{C}_{4}$ cytosine-like amine group of gemcitabine.

Phase-II Synthesis Scheme for Covalent Gemcitabine-( $C_{4}$-amide)-[anti-HER2/neu] Immunochemotherapeutic Utilizing a UV-Photoactivated Gemcitabine Intermediate-Immunoglobulin fractions of anti-HER2/neu (1.5 mg, $1.0 \times 10^{-5}$ mmoles) in buffer (PBS: phosphate $0.1, \mathrm{NaCl} 0.15 \mathrm{M}$, EDTA $10 \mathrm{mM}, \mathrm{pH} 7.3$ ) were com- 

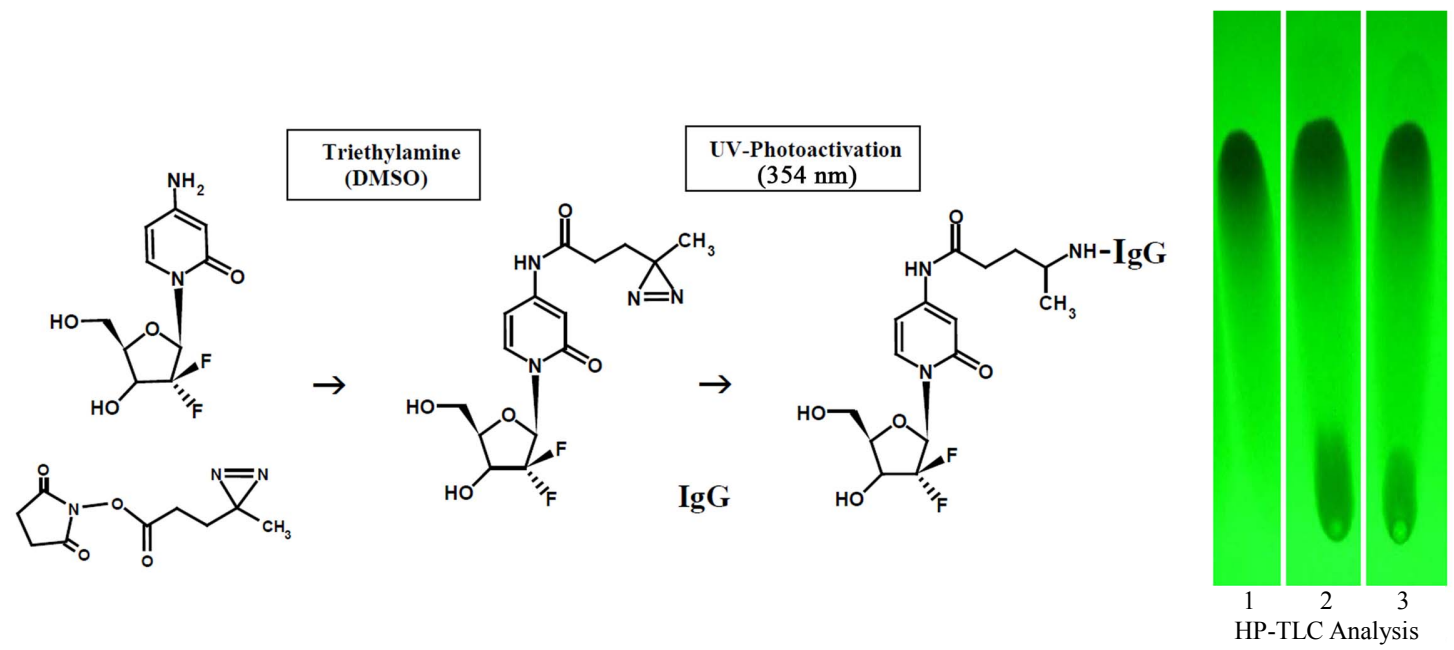

Figure 1. Schematic illustration of the organic chemistry reaction schemes utilized in the 2-phase synthesis regimen for gemcitabine-( $\mathrm{C}_{4}$-amide)-[anti-HER2/neu]. Legends for Reactions: (Phase-I) creation of a covalent amide bond at the $\mathrm{C}_{4}$ cytosine-like monoamine of gemcitabine and the ester group of succinimidyl 4,4-azipentanoate resulting in the creation of a covalent UV-photoactivated gemcitabine- $\left(\mathrm{C}_{4}\right.$-amide $)$ intermediate. The reaction results in the liberation of the succinimide "leaving" complex. (Phase-II) creation of a covalent bond between the UV-photoactivated gemcitabine- $\left(\mathrm{C}_{4}\right.$-amide $)$ intermediate and chemical groups within the amino acid sequence of anti-HER2/neu monoclonal immunoglobulin initiated by exposure to UV light (354 nm). Legends for HP-TLC Analysis: Reaction of the $N$-hydroxy-succinymide groups of disuccinimidyl glutarate with the $\mathrm{C}_{4}$ cytosine like "ring amine" of gemcitabine. (Lane-1) gemcitabine reference control; (Lane-2) gemcitabine reacted with disuccinmidyl glutarate in DMSO with Tri-ethylamide at $50 \mathrm{mM}$ final concentration; and (Lane-3) gemcitabine reacted with disuccinmidyl glutarate in DMSO and $\mathrm{ddH}_{2} \mathrm{O}(2: 1 \mathrm{v} / \mathrm{v})$. Reaction products were developed by silica gel HP-TLC using a mobile phase of propanol/ethanol (80:20 v/v) and images visualized under UV light (254 nm).

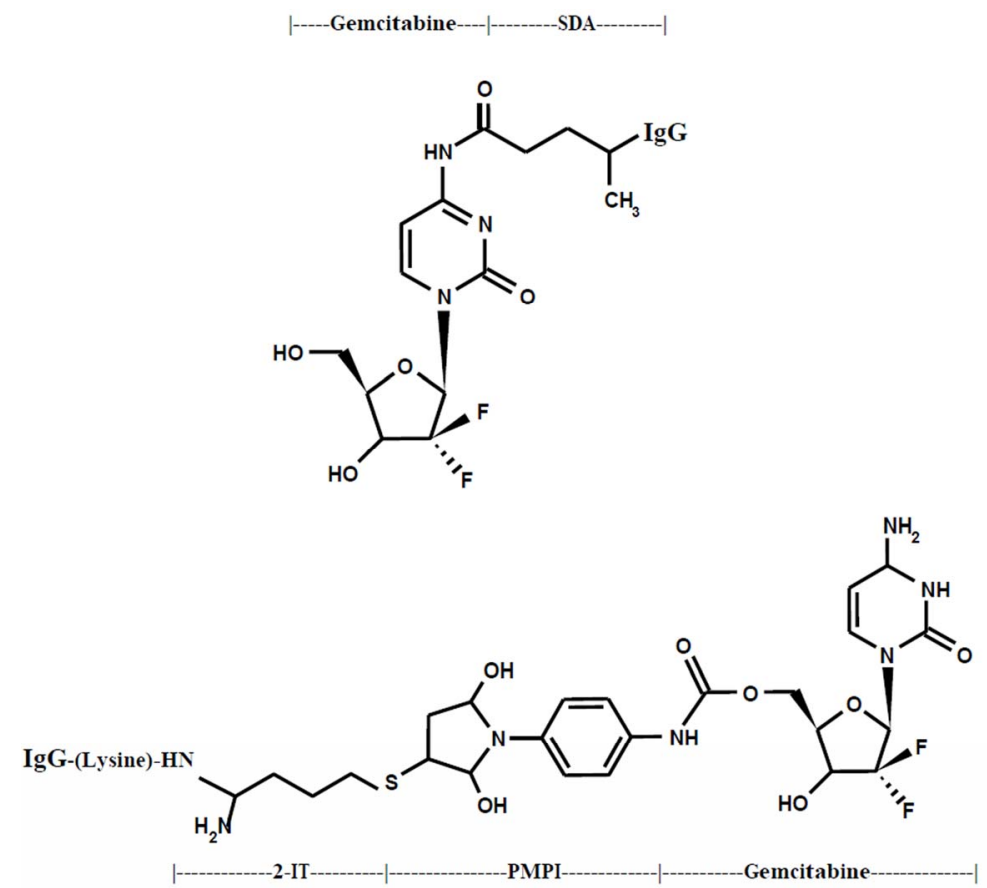

Figure 2. Molecular design and chemical composition of two covalent gemcitabine-immunochemotherapeutics. Legends: (Top-Panel) Gemcitabine-(C4-amide)-[anti-HER2/neu] synthesized using a 2-stage organic chemistry reaction scheme that initially creates a covalent bond at the $\mathrm{C}_{4}$ cytosine-like amine group of gemcitabine. (Bottom-Panel) Gemcitabine-( $\mathrm{C}_{5}-\mathrm{me}$ thylcarbamate)-[anti-HER2/neu] synthesized using a 3-stage organic chemistry reaction scheme that formed covalent bonds at the chemotherapeutic $\mathrm{C}_{5}$-methylhydroxy group and at/to thiolated lysine $\alpha$-amine groups residing within the amino acid sequence of anti-HER2/neu monoclonal immunoglobulin fractions. 
bined at a 1:10 molar-ratio with the UV-photoactivated gemcitabine- $\left(\mathrm{C}_{4}\right.$-amide $)$ intermediate (Phase-1 end prod$u c t$ ) and allowed to gently mix by constant stirring for 5 minutes at $25^{\circ} \mathrm{C}$ in the dark. The photoactivated group of the gemcitabine- $\left(\mathrm{C}_{4}\right.$-amide $)$ intermediate was then reacted with side chains of amino acid residues within the sequence of anti-HER2/neu monoclonal immunoglobulin during a 15 minute exposure to UV light at $354 \mathrm{~nm}$ (reagent activation range $320-370 \mathrm{~nm}$ ) in combination with constant gentle stirring (Figures 1 and 2). Residual chemotherapeutic was removed from the covalent gemcitabine- $\left(\mathrm{C}_{4}\right.$-amide)-[anti-HER2/neu] immunochemotherapeutic applying micro-scale column chromatography following pre-equilibration of exchange media with PBS (phosphate $0.1, \mathrm{NaCl} 0.15 \mathrm{M}, \mathrm{pH} 7.3$ ).

\subsection{Gemcitabine-( $\mathrm{C}_{5}$-methylcarbamate $)$ - [anti-HER2/neu] Immunochemotherapeutic Synthesis}

Phase-I: Immunoglobulin Thiolation at Lysine $\varepsilon$-Amine Groups-A purified fraction of monoclonal immunoglobulin with binding-avidity specifically for human HER2/ neu (ErbB-2, CD 340) was utilized for the semi-synthesis of gemcitabine- $\left(\mathrm{C}_{5}\right.$-methylcarbamate)-[anti-HER2/neu] [36]. Desiccated anti-HER2/neu monoclonal immunoglobulin $(1.5 \mathrm{mg})$ was combined with 2-imino-thiolane (2-IT: $6.5 \mathrm{mM}$ final concentration) in PBS $(0.1 \mathrm{M}, \mathrm{pH}$ $8.0,250 \mu \mathrm{l})$ and incubated at $25^{\circ} \mathrm{C}$ for 1.5 hours in combination with simultaneous constant gentle stirring [8, 50-52]. Thiolated anti-HER2/neu monoclonal immunoglobulin was then buffer exchanged into PBS-EDTA (phosphate $0.1, \mathrm{NaCl} 0.15 \mathrm{M}$, EDTA $10 \mathrm{mM}, \mathrm{pH} 7.3$ ) using micro-scale column chromatography. Moles of reduced sulfhydryl groups covalently introduced into anti-HER2/neu monoclonal immunoglobulin was measured with a 5,5'-dithiobis-(2-nitrobenzoic acid (DTNB reagent) based assay. The average number of thiolated lysine $\varepsilon$-amine groups introduced into anti-HER2/neu fractions $(\mathrm{R}-\mathrm{SH} / \mathrm{IgG})$ was $3: 1$ based on results with 2 -IT reagent.

Phase-II: Synthesis of Gemcitabine- $\left(C_{5}\right.$-methylcarbamate)-PMPI Sulfhydryl Reactive Intermediate-Gemcitabine in DMSO $\left(0.738 \mathrm{mg}, 2.80 \times 10^{-3}\right.$ mmoles $)$ was combined at a 5:1 molar ratio with $\mathrm{N}$ - $[p$-maleimidophenyl]-isocyanate (PMPI: $0.120 \mathrm{mg}, 5.60 \times 10^{-4}$ mmoles) [36,53-55] and allowed to mix by constant gentle stirring at $25^{\circ} \mathrm{C}$ for 3.5 hours. Under these conditions the PMPI isocyanate moiety exclusively reacts with hydroxyl (R$\mathrm{OH})$ groups and preferentially creates a carbamate covalent bond at the terminal $\mathrm{C}_{5}$-methylhydroxy group of gemcitabine [36,40,56-61]. The highly selective reaction is reportedly complete within 2 hours under the conditions applied as described. Gemcitabine was formulated at a large molar excess to deplete un-reacted PMPI and maximize synthesis of the sulfhydryl-reactive maleimide intermediate.

Phase-III: Covalent Reaction of Gemcitabine- $\left(C_{5^{-}}\right.$ methylcarbamate)-PMPI Intermediate with Thiolated Immunoglobulin-The gemcitabine-( $\mathrm{C}_{5}$-methylcarbamate $)$-PMPI intermediate with a maleimide moiety that exclusively reacts with reduced sulfhydryl (R-SH) groups was combined at a 1.5:1 molar ratio with thiolated terminal lysine $\varepsilon$-amines in anti-HER2/neu monoclonal immunoglobulin fractions (PBS-EDTA: phosphate $0.1, \mathrm{NaCl} 0.15 \mathrm{M}$, EDTA $10 \mathrm{mM}, \mathrm{pH} \mathrm{7.3)}$ and the formulation mixture incubated with constant stirring at $25^{\circ} \mathrm{C}$ for 2 hours $[2,3,7,9,25,26,28,36,53,62-66]$. Similar synthesis strategies in concept have previously been applied to produce covalent anthracycline immunochemotherapeutic preparations $[7,8,50,51,67,68]$. Because of the selective characteristics of the synthesis scheme employed to produce the sulfhydryl-reactive gemcitabine- $\left(\mathrm{C}_{5}\right.$-methylcarbamate $)$ PMPI intermediate and the limited duration of chemical stability associated with it's maleimide moiety in aqueous buffers, the preparation was directly mixed with thiolated anti-HER2/neu fractions [7,36,51]. Residual gemcitabine was removed from the final covalent gemcitabine- $\left(\mathrm{C}_{5}\right.$-methylcarbamate)-[anti-HER2/neu] immunochemotherapeutic end-product applying microscale column chromatography following pre-equilibration of exchange media with PBS (phosphate $0.1, \mathrm{NaCl} 0.15 \mathrm{M}$, $\mathrm{pH}$ 7.3) yielding a homogenous purified preparation (Figure 2).

\subsection{Analysis and Property Characteristics}

General Analysis-Quantitation of the amount of noncovalently bound gemcitabine contained within covalent gemcitabine-( $\mathrm{C}_{4}$-amide $)$-[anti-HER2/neu] and gemcitabine- $\left(\mathrm{C}_{5}\right.$-methylcarbamate $)$-[anti-HER2/neu] im-munochemotherapeutics following separation by column chromatography was determined by measured absorbance at $265-268 \mathrm{~nm}[69,70]$ for the resulting supernatant after precipitation of gemcitabine-immuno-chemotherapeutics with methanol:acetonitrile $(1: 9 \mathrm{v} / \mathrm{v})$.

In contrast to the anthracyclines, $[7,71,72]$ gemcitabine can not be measured directly within covalent immunochemotherapeutic preparations by spectrophotometric absorption [36]. Alternatively it is possible to calculate the amount of gemcitabine that has been covalent incurporated into immunochemotherapeutics by measuring residual unbound gemcitabine before and after the Phase II reaction or by measuring the difference in non-chemotherapeutic-occupied sites associated with either amine or reduced sulfhydryl groups within anti-HER2/neu monoclonal immunoglobulin compared to gemcitabine-immunochemotherapeutics $[36,51,52]$. 
Determination of the gemcitabine molar-incorporation-Index and gemcitabine molar-equivalent-concentrations for gemcitabine- $\left(\mathrm{C}_{5}\right.$-methylcarbamate)-[antiHER2/ $n e u$ ] were calculated using measurements for the relative difference in moles of reduced sulfhydryl groups (e.g. R-SH: cystine amino acid residues and sulfhydryl groups introduced with Traut's reagent) contained within thiolated anti-HER2/neu fractions relative to the covalent gemcitabine-immuno-chemotherapeutic following separation by column chromatography $[36,51,52]$. Reduced sulfhydryl groups were measure by combining anti-HER2/ $n e u$ or gemcitabine $\left(\mathrm{C}_{5}\right.$-methylcarbamate)-[anti-HER2/ $n e u]$ in phosphate buffered saline $(0.1 \mathrm{M}, \mathrm{pH} 7.4)$ with 5,5'-dithiobis-(2-nitrobenzoic acid) formulated in sodium phosphate-EDTA buffer (DTNB: $78 \mu \mathrm{g} / \mathrm{ml}$ with EDTA 1 $\mathrm{mM}$ in $0.1 \mathrm{M}$ sodium phosphate buffer $0.1 \mathrm{M}$, $\mathrm{pH} 8.0$ ). Spectrophotometric absorbance of mixtures formulated at $1: 1 \mathrm{v} / \mathrm{v}$ (e.g. $250 \mu \mathrm{l}$ each) was measured at $412 \mathrm{~nm}$ following incubation at $25^{\circ} \mathrm{C}$ for 15 minutes. The amount and concentration of sulfhydryl groups was then calculated utilizing a linearized standard curve generated with reference control solutions of cysteine $\mathrm{HCl}$ monohydrate formulated at known concentrations (molar extinction coefficient: $14,150 \mathrm{M}^{-1} \cdot \mathrm{cm}^{-1}$ ).

Determination of the immunoglobulin concentration for covalent gemcitabine-(C4-amide)-[anti-HER2/neu] and gemcitabine-( $\mathrm{C}_{5}$-methylcarbamate)-[anti-HER $2 /$ neu] immunochemotherapeutics was determined by measuring spectrophotometric absorbance at $280 \mathrm{~nm}$ in combinations with utilizing a $235 \mathrm{~nm}-\mathrm{vs}-280 \mathrm{~nm}$ standardized reference curve in order to accommodate for any potential absorption profile over-lap at $280 \mathrm{~nm}$ between gemcitabine and immunoglobulin.

Mass/Size-Dependent Separation of Gemcitabine-Immunochemotherapeutics by Non-Reducing SDS-PAGECovalent gemcitabine-( $\mathrm{C}_{4}$-amide)-[anti-HER2/neu] and gemcitabine $\left(\mathrm{C}_{5}\right.$-methylcarbamate)-[anti-HER2/neu] immunochemotherapeutics in addition to a anti-HER2/neu immunoglobulin reference control fraction were adjusted to a standardized protein concentration of 60 $\mu \mathrm{g} / \mathrm{ml}$ and then combined $50 / 50 \mathrm{v} / \mathrm{v}$ with conventional SDS-PAGE sample preparation buffer (Tris/glycerol/ bromphenyl blue/SDS) formulated without 2-mercaptoethanol or boiling. Each covalent gemcitabine immunochemotherapeutic, the reference control immunoglobulin fraction $(0.9 \mu \mathrm{g} / \mathrm{well})$ and a mixture of prestained reference control molecular weight markers were then developed by non-reducing SDS-PAGE (11\% acrylamide) performed at $100 \mathrm{~V}$ constant voltage at $3^{\circ} \mathrm{C}$ for 2.5 hours.

Immunodetection Analyses-Covalent gemcitabine- $\left(\mathrm{C}_{4}\right.$ amide)-[anti-HER2/neu] and gemcitabine $\left(\mathrm{C}_{5}\right.$-methylcarbamate)-[anti-HER2/neu] immunochemo-therapeutics following mass/size-dependent separation by non-reducing SDS-PAGE were equilibrated in tank buffer devoid of methanol. Mass/size-separated gemcitabine and anthracycline anti-HER2/neu immunochemotherapeutics contained in acrylamide SDS-PAGE gels were then transferred laterally onto sheets of nitrocellulose membrane at 20 volts (constant voltage) for 16 hours at $2^{\circ} \mathrm{C}$ to $3^{\circ} \mathrm{C}$ with the transfer manifold packed in crushed ice.

Nitrocellulose membranes with laterally-transferred immunochemotherapeutics were then equilibrated in Trisbuffered saline (TBS: Tris $\mathrm{HCl} 0.1 \mathrm{M}, \mathrm{NaCl} 150 \mathrm{mM}$, pH $7.5,40 \mathrm{ml})$ at $4^{\circ} \mathrm{C}$ for 15 minutes followed by incubation in TBS blocking buffer solution (Tris $0.1 \mathrm{M}, \mathrm{pH} 7.4,40$ $\mathrm{ml}$ ) containing bovine serum albumin (5\%) for 16 hours at $2^{\circ} \mathrm{C}$ to $3^{\circ} \mathrm{C}$ applied in combination with gentle horizontal agitation. Prior to further processing, nitrocellulose membranes were vigorously rinsed in Tris buffered saline (Tris 0.1 M, pH 7.4, $40 \mathrm{ml}, \mathrm{n}=3 \mathrm{x}$ ).

Rinsed BSA-blocked nitrocellulose membranes developed for immunodetection (Western-blot) analyses were incubated with biotinylated goat anti-murine $\mathrm{IgG}$ (1:10,000 dilution) at $4^{\circ} \mathrm{C}$ for 18 hours applied in combination with gentle horizontal agitation. Nitrocellulose membranes were then vigorously rinsed in TBS $(\mathrm{pH} 7.4$, $4^{\circ} \mathrm{C}, 50 \mathrm{ml}, \mathrm{n}=3$ ) followed by incubation in blocking buffer (Tris $0.1 \mathrm{M}, \mathrm{pH} 7.4$, with BSA $5 \%, 40 \mathrm{ml}$ ). Blocking buffer was decanted from nitrocellulose membrane blots and then rinsed in TBS $\left(\mathrm{pH} \mathrm{7.4,} 4^{\circ} \mathrm{C}, 50 \mathrm{ml}, \mathrm{n}\right.$ $=3)$ before incubation with strepavidin-HRPO $\left(1: 100,000\right.$ dilution) at $4^{\circ} \mathrm{C}$ for 2 hours applied in combination with gentle horizontal agitation. Prior to chemiluminescent development nitrocellulose membranes were vigorously rinsed in Tris buffered saline (Tris $0.1 \mathrm{M}, \mathrm{pH}$ $7.4,40 \mathrm{ml}, \mathrm{n}=3$ ). Development of nitrocellulose membranes by chemiluminescent autoradiography following processing with conjugated HRPO-strepavidin required incubation in $\mathrm{HRPO}$ chemiluminescent substrate $\left(25^{\circ} \mathrm{C}, 5\right.$ to 10 mins.). Autoradiographic images were acquired by exposing radiographic film (Kodak BioMax XAR) to nitrocellulose membranes sealed in transparent ultraclear re-sealable plastic bags.

Mammary Adenocarcinoma Tissue Culture Cell Culture-The chemotherapeutic-resistant (SKBr-3) human mammary adenocarcinoma cell line was utilized as an ex-vivo neoplasia model. Mammary adenocarcinoma (SKBr-3) characteristically over-expresses epidermal growth factor receptor 1 (EGFR, ErbB-1, HER1) and highly over-expresses epidermal growth factor receptor 2 (EGFR2, HER2/neu, ErbB-2, CD340, p185) at $2.2 \times$ $10^{5} /$ cell and $1 \times 10^{6} /$ cell respectively.

Populations of the mammary adenocarcinoma ( $\mathrm{SKBr}-3$ ) were propagated in $150-\mathrm{cc} 2$ tissue culture flasks containing McCoy's 5a Modified Medium supplemented 
with fetal bovine serum $(10 \% \mathrm{v} / \mathrm{v})$ and penicillin-streptomycin at a temperature of $37^{\circ} \mathrm{C}$ under a gas atmosphere of air $(95 \%)$ and carbon dioxide $\left(5 \% \mathrm{CO}_{2}\right)$. Tissue culture media was not supplemented with growth factors, growth hormones or other growth stimulants of any type. Investigations were performed using mammary adenocarcinoma (SKBr-3) monolayer populations at a $>85 \%$ level of confluency.

Cell-ELISA Total Membrane-Bound Immunoglobulin Assay-Cell suspensions of mammary adenocarcinoma (SKBr-3) were seeded into 96-well microtiter plates in aliquots of $2 \times 10^{5}$ cells/well and allowed to form a confluence adherent monolayer over a period of 48 hours. The growth media contents of individual wells was then removed manually by pipette and serially rinsed $(n=3)$ with PBS followed by stabilization of adherent cellular monolayers onto the plastic surface of 96-well plates with paraformaldehyde (4\% in PBS, 15 minutes). Stabilized mammary adenocarcinoma ( $\mathrm{SKBr}-3)$ monolayers were then incubated with gemcitabine-( $\mathrm{C}_{4}$-amide)-[antiHER2/neu] or gemcitabine $\left(\mathrm{C}_{5}\right.$-methylcarbamate)-[antiHER2/neu] immunoconjugates formulated at gradient concentrations of $0.1,0.25,0.5,1.0,5.0$ and $10 \mu \mathrm{g} / \mathrm{ml}$ in tissue culture growth media $(200 \mu 1 /$ well). Direct contact incubation between mammary adenocarcinoma (SKBr-3) cellular monolayers and gemcitabine-( $\mathrm{C}_{4}$-amide $)$-[antiHER2/neu] or gemcitabine-( $\mathrm{C}_{5}$-methylcarbamate)-[antiHER2/neu] at $37^{\circ} \mathrm{C}$ was performed over an incubation period of 3-hours using a gas atmosphere of air (95\%) and carbon dioxide $\left(5 \% \mathrm{CO}_{2}\right)$. Following serial rinsings with PBS $(n=3)$, development of stabilized mammary adenocarcinoma (SKBr-3) monolayers entailed incubation with $\beta$-galactosidase conjugated goat anti-mouse $\operatorname{IgG}\left(1: 500\right.$ dilution) for 2 hours at $25^{\circ} \mathrm{C}$ with residual unbound immunoglobulin removed by serial rinsing with PBS $(\mathrm{n}=3)$. Final cell ELISA development required serial rinsing $(\mathrm{n}=3)$ of stabilized cellular monolayers with PBS followed by incubation with nitrophenyl- $\beta$ D-galactopyranoside substrate $(100 \mu \mathrm{l} /$ well of ONPG formulated fresh at $0.9 \mathrm{mg} / \mathrm{ml}$ in PBS pH 7.2 containing $\mathrm{MgCl}_{2} 10 \mathrm{mM}$, and 2-mercaptoethanol $0.1 \mathrm{M}$ ). Absorbance within each individual well was measured at 410 $\mathrm{nm}(630 \mathrm{~nm}$ reference wavelength) after incubation at $37^{\circ} \mathrm{C}$ for a period of 15 minutes.

Cell Vitality Stain-Based Assay for Measuring Cytotoxic Anti-Neoplastic Potency-Individual preparations of gemcitabine-( $\mathrm{C}_{4}$-amide)-[anti-HER2/neu] and gemcitabine-( $\mathrm{C}_{5}$-methylcarbamate)-[anti-HER2/neu] were formulated in growth media at standardized chemotherapeutic-equivalent concentrations of $10^{-10}, 10^{-9}, 10^{-8}, 10^{-7}$, and $10^{-6} \mathrm{M}$ (final concentration). Each chemotherapeutic-equivalent concentration of covalent immunochemotherapeutic was then transferred in triplicate into 96-well microtiter platesm containing mammary adenocarcinoma (SKBr-3) monolayers (growth media $200 \mu \mathrm{l} /$ well). Covalent gemcitabine immunochemotherapeutics where then incubated in direct contact with monolayer mammary adenocarcinoma SKBr-3 populations for a period of 182hours at $\left(37^{\circ} \mathrm{C}\right.$ under a gas atmosphere of air $(95 \%)$ and carbon dioxide $/ \mathrm{CO}_{2}(5 \%)$. Following the initial 72-hour incubation period, mammary adenocarcinoma ( $\mathrm{SKBr}-3)$ populations were replenished with fresh tissue culture media with or without covalent gemcitabine-immunochemotherapeutics.

Cytotoxic potencies for gemcitabine- $\left(\mathrm{C}_{4}\right.$-amide $)$-[antiHER2/neu] and gemcitabine-( $\mathrm{C}_{5}$-methylcarbamate $)$ [antiHER 2/neu] were measured by removing all contents within the 96 -well microtiter plates manually by pipette followed by serial rinsing of monolayers $(n=3)$ with PBS and incubation with 3-[4,5-dimethylthiazol-2-yl] -2,5-diphenyl tetrazolium bromide vitality stain reagent formulated in RPMI-1640 growth media devoid of $\mathrm{pH}$ indicator or bovine fetal calf serum (MTT: $5 \mathrm{mg} / \mathrm{ml}$ ). During an incubation period of $3-4$ hours at $37^{\circ} \mathrm{C}$ under a gas atmosphere of air $(95 \%)$ and carbon dioxide $(5 \%$ $\mathrm{CO}_{2}$ ) the enzyme mitochondrial succinate dehydrogenase was allowed to convert the MTT vitality stain reagent to navy-blue formazone crystals within the cytosol of mammary adenocarcinoma (SKBr-3) populations. Contents of the 96-well microtiter plate was then removed, followed by serial rinsing with PBS $(n=3)$. The resulting blue intracellular formzone crystals were dissolved with DMSO (300 $\mu 1 /$ well) and then the spectrophotometric absorbance of the blue-colored supernantant measured at $570 \mathrm{~nm}$ using a computer integrated microtiter plate reader.

\section{Results}

Molar-Incorporation Index-Size-separation of covalent immunochemotherapeutics like gemcitabine-( $\mathrm{C}_{4}$-amide $)$ [anti-HER2/neu] and gemcitabine- $\left(\mathrm{C}_{5}\right.$-methylcarbamate)[anti-HER2/neu] by micro-scale exchange column chromatography consistently yields preparations that contain $<4.0 \%$ of residual chemotherapeutic that is not covalently bound to the immunoglobulin fraction $[7,36,71,72]$. Small residual amounts of non-covalently bound chemotherapeutic remaining within covalent immunochemotherapeutic preparations is generally considered to not be available for further removal through any additional sequential column chromatography separations [73]. The calculated estimate of the molar-incorporation-index for the covalent gemcitabine-( $\mathrm{C}_{4}$-amide)-[anti-HER2/neu] immunochemotherapeutic was 2.78 utilizing the organic chemistry reaction scheme that forms an amide bond at the $\mathrm{C}_{4}$ cytosine-like amine of gemcitabine resulting in the initial synthesis of the UV-photoactivated gemcitabine- 
$\left(\mathrm{C}_{4}\right.$-amide) intermediate (Figures 1 and 2$)$. The molar-incorporation-ration of 2.78-to-1 for gemcitabine$\left(\mathrm{C}_{4}\right.$-amide)-[anti-HER2/neu] was relatively larger than the 1.1-to-1 gemcitabine molar-incorporation-index attained during the synthesis of gemcitabine- $\left(\mathrm{C}_{5}-\right.$ me-thylcarbamate)-[anti-HER2/neu] [36].

Molecular Weight Profile Analysis-Mass/size separation of covalent gemcitabine-( $\mathrm{C}_{4}$-amide $)$-[anti-HER2/ $n e u]$ and gemcitabine- $\left(\mathrm{C}_{5}\right.$-methylcarbamate $)$-[anti-HER2/ $n e u$ immunochemotherapeutics by SDS-PAGE in combination with immunodetection analysis (Western blot) and chemiluminescent autoradiography recognized a single primary condensed band of $150-\mathrm{kDa}$ between a molecular weight range of $5.0-\mathrm{kDa}$ to $450-\mathrm{kDa}$ (Figure 3) Patterns of low-molecular-weight fragmentation (proteolytic/hydrolytic degradation) or large-molecular-weight immunoglobulin polymerization were not detected (Figure 3). The observed molecular weight of $150-\mathrm{kDa}$ for both gemcitabine-( $\mathrm{C}_{4}$-amide $)$-[anti-HER2/neu] and gemcitabine- $\left(\mathrm{C}_{5}\right.$-methylcarbamate $)$-[anti-HER2/neu] directly corresponds with the known molecular weight/mass of reference control anti-HER2/neu monoclonal immunoglobulin fractions (Figure 3). Analogous results have been reported for similar covalent immunochemotherapeutics [2,7,36,71,72,74].

Cell-Binding Analysis-Total bound immunoglobulin in the form of gemcitabine-( $\mathrm{C}_{4}$-amide $)$-[anti-HER2/neu] or gemcitabine- $\left(\mathrm{C}_{5}\right.$-methylcarbamate $)$-[anti-HER2/neu] on the external surface membrane of adherent mammary adenocarcinoma (SKBr-3) populations was measured by cell-ELISA (Figure 4). Greater total membrane-bound gemcitabine-( $\mathrm{C}_{4}$-amide $)$-[anti-HER2/neu] was detected with progressive increases in standardized total immu-

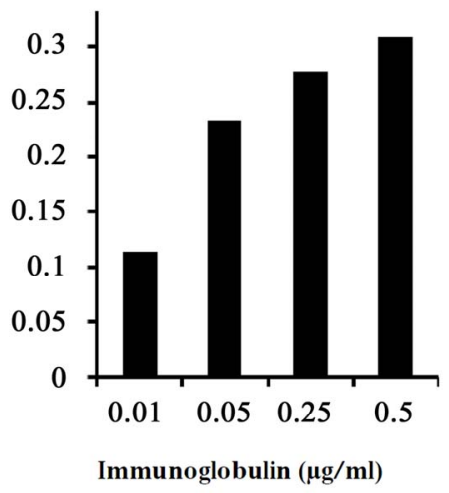

gemcitabine- $\left(\mathrm{C}_{4}-\mathrm{NH}_{2}\right)-[$ anti-HER2/neu]

noglobulin-equivalent concentrations formulated at 0.010 , $0.025,0.050,0.250$, and $0.500 \mu \mathrm{g} / \mathrm{ml}$ (Figure 4). In order to detect elevations in total membrane-bound gemcitabine- $\left(\mathrm{C}_{5}\right.$-methylcarbamate)-[anti-HER2/neu] standardized total immunoglobulin-equivalent concentrations had to alternatively be formulated at $0.5,1.0,5.0$ and 10.0 $\mu \mathrm{g} / \mathrm{ml}$ (Figure 4). Collectively each of these sets of cell-ELISA findings serve to validate the retained selective binding-avidity of gemcitabine- $\left(\mathrm{C}_{4}\right.$-amide $)$-[antiHER2/neu] and gemcitabine- $\left(\mathrm{C}_{5}\right.$-methylcarbamate $)$ [anti-HER2/neu] for external membrane HER2/neu re-

150 kDa

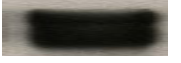

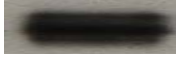

2

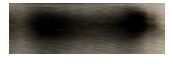

3
Figure 3. Characterization of the major molecular weight profile for covalent gemcitabine-( $\mathrm{C}_{4}$-amide $)$-[anti-HER2/neu] and gemcitabine-( $\mathrm{C}_{5}$-methylcarbamate $)$-[anti-HER2/neu] immunochemotherapeutics compared to anti-HER2/neu monoclonal immunoglobulin. Legends: (Lane-1) murine antihuman HER2/neu monoclonal immunoglobulin reference control; (Lane-2) covalent gemcitabine-( $\mathrm{C}_{4}$-amide)-[antiHER2/neu] immunochemotherapeutic; and (Lane-3) covalent gemcitabine-( $\mathrm{C}_{5}$-methylcarbamate)-[anti-HER2/neu] immunochemotherapeutic. Covalent gemcitabine immunochemotherapeutics or anti-HER2/ neu monoclonal immunogloublin were size-separated by non-reducing SDS-PAGE followed by lateral transfer onto sheets of nitrocellulose membrane to facilitate detection with biotinylated goat anti-mouse IgG immunoglobulin. Subsequent analysis entailed incubation of nitrocellulose membranes with strepavidin-HRPO in combination with the use of a HRPO chemiluminescent substrate for acquisition of autoradiography images.

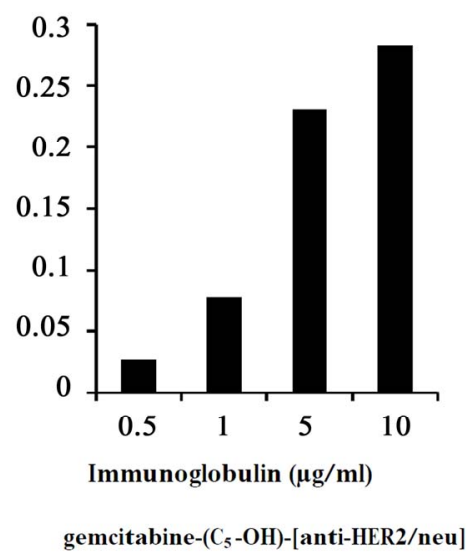

Figure 4. Detection of total anti-HER2/neu immunoglobulin in the form of gemcitabine-( $\mathrm{C}_{4}$-amide)-[anti-HER2/neu] and gemcitabine-( $\mathrm{C}_{5}$-methylcarbamate)-[anti-HER2/neu] bound to the exterior surface membrane of chemotherapeutic-resistant mammary adenocarcinoma (SKBr-3). Legends: (Left-Panel) gemcitabine-( $\mathrm{C}_{4}$-amide)-[anti-HER2/neu]; and (Right-Panel) gemcitabine-( $\mathrm{C}_{5}$-methylcarbamate)-[anti-HER2/neu]. Monolayer populations of mammary adenocarcinoma (SKBr-3) were incubated with the covalent gemcitabine-( $\mathrm{C}_{4}$-amide $)$-[anti-HER2/neu] or gemcitabine- $\left(\mathrm{C}_{5}\right.$-methylcarbamate $)$-[anti-HER2/neu] immunochemotherapeutics over a 4-hour period and total immunoglobulin bound to the exterior surface membrane was then measured by cell-ELISA. 
ceptor sites highly over-expressed at $1 \times 106 /$ cell on the exterior surface membrane of mammary adenocarcinoma (SKBr-3) populations (Figure 4) [36].

Cytotoxic Anti-Neoplastic Potency Analysis-Gemcitabine( $\mathrm{C}_{4}$-amide)-[anti-HER2/neu $]$ and gemcitabine-( $\mathrm{C}_{5}$-methylcarbamate)-[anti-HER $2 / n e u$ ] exerted $41.1 \%$ and $30.8 \%$ maximum selective "targeted" cytotoxic anti-neoplastic potency (58.9\% and $69.2 \%$ residual survival) against chemotherapeutic-resistant mammary adenocarcinoma ( $\mathrm{SKBr}-3)$ at the gemcitabine-equivalent concentration of $10^{-6} \mathrm{M}$ respectively (Figures 5-7). Profiles for the cytoto$x i c$ anti-neoplastic potency of gemcitabine- $\left(\mathrm{C}_{4}\right.$-amide $)$ [anti-HER2/neu] and gemcitabine-( $\mathrm{C}_{5}$-methylcarbamate)[anti- HER2/neu] after a 182-hour incubation period were highly analogous to gemcitabine chemotherapeutic following a 72-hour incubation period at the gemcitabineequivalent concentrations of $10^{-10} \mathrm{M}, 10^{-9} \mathrm{M}, 10^{-8} \mathrm{M}$, $10^{-7} \mathrm{M}$ and $10^{-6} \mathrm{M}$ (Figures 5 and 6). The cytotoxic anti-neoplastic potency of gemcitabine-( $\mathrm{C}_{4}$-amide)-[antiHER2/neu], gemcitabine-( $\mathrm{C}_{5}$-methylcarbamate)-[anti-HER2/ $n e u]$ and gemcitabine after a 182-hour incubation period were essentially equivalent at the gemcitabine-equivalent concentrations of $10^{-10} \mathrm{M}$ and $10^{-9} \mathrm{M}$ but not at $10^{-7} \mathrm{M}$ or $10^{-6} \mathrm{M}$ (Figures 5 and 7) [36]. Mean maximum cytotoxic anti-neoplastic potencies for gemcitabine- $\left(\mathrm{C}_{4}\right.$-amide $)$ [anti-HER2/neu] at 182-hours, gemcitabine at 72-hours, and gemcitabine at 182 -hours were $41.1 \%, 48.0 \%$ and $88.3 \%(58.9 \%, 52.0 \%$ and $11.7 \%$ residual survival) at the gemcitabine-equivalent concentration of $10-\mathrm{M}$ respectively (Figure 5). Gemcitabine-( $\mathrm{C}_{4}$-amide)-[anti-HER2/ $n e u$ ] and gemcitabin-( $\mathrm{C}_{5}$-methylcarbamate)-[anti-HER2/ $n e u]$ immunochemotherapeutic both exerted profiles for cytotoxic anti-neoplastic potency against chemotherapeutic mammary adenocarcinoma (SKBr-3) that were similar to epirubicin-(C3-amide)-[anti-HER2/neu] but only at the chemotherapeutic-equivalent concentrations of $10^{-10} \mathrm{M}, 10^{-9} \mathrm{M}$ and $10^{-8} \mathrm{M}$ respectively (Figure 8) [71]. The level of cytotoxic anti-neoplastic potency for epirubicin-(C3-amide)-[anti-HER2/neu] was substantially higher at the chemotherapeutic-equivalent concentrations of $10^{-7} \mathrm{M}$ and $10^{-6} \mathrm{M}$ after a 72 -hour incubation period (Figure 8). Mean maximum levels of anti-neoplastic potency for gemcitabine-( $\mathrm{C}_{4}$-amide)-[anti-HER2/neu], gemcitabin-( $\mathrm{C}_{5}$-methylcarbamate)-[anti-HER2/neu] and epiribicin (C13-imino)-[anti-HER2/neu] were 41.1\% (182hours), $30.8 \%$ (182-hours) and $88.5 \%$ (72-hours) at the chemotherapeuticequivalent concentration of $10^{-6} \mathrm{M}$ respectively (Figures 5-8).

Comparison of the cytotoxic anti-neoplastic potency of gemcitabine-( $\mathrm{C}_{4}$-amide)-[anti-HER2/neu] and gemcitabine$\left(\mathrm{C}_{5}\right.$-methylcarbamate)-[anti-HER2/neu] as a function of immunoglobulin-equivalent concentrations (standardized anti-HER2/neu content) and gemcitabine molarincorpora- tion-index detected distinct differences between the two covalent gemcitabine-immunochemo-therapeutics (Figure 9). Given this perspective, gemcitabine $\left(\mathrm{C}_{4}\right.$-amide)-[antiHER2/neu] and gemcitabine-( $\mathrm{C}_{5}$-methylcarbamate)-[antiHER 2/neu ] each exerted an equivalent level of cytotoxic anti-neoplastic potency against chemotherapeutic-resistant mammary adenocarcinoma (SKBr-3) at immunoglobulin-equivalent concentrations of $6.9 \times 10^{-8} \mathrm{M}$ and $9.1 \times$ $10^{-9} \mathrm{M}$ respectively (Figure 9). Based on these calculations, gemcitabine-( $\mathrm{C}_{4}$-amide $)$-[anti-HER2/ neu] was approximately 7.6 -fold more potent than gemcitabine- $\left(\mathrm{C}_{5^{-}}\right.$ methylcarbamate)-[anti-HER2/neu] at a cytotoxic antineoplastic potency level of approximately $30 \%$ when standardized as a function of immunoglobulin-equivalent concentration (Figure 9). Monoclonal anti-HER2/neu [7,36,71,72] and anti-EGFR [7] immunoglobulin fractions alone between 0-to-182-hours do not exert detectable levels of ex-vivo cytotoxic anti-neoplastic potency against chemotherapeutic-resistant mam- mary adenocarcinoma (SKBr-3) which is in direct accord with previous investigations (Figure 10) [7,28,29,32, 74,75].

\section{Discussion}

The creation of a synthetic covalent bond between gemcitabine and monoclonal immunoglobulin, immuno-

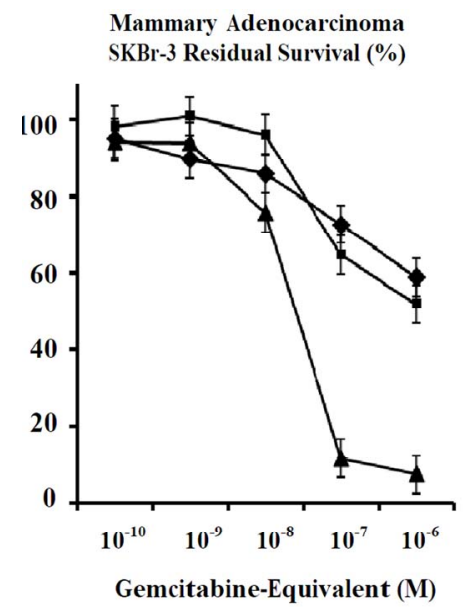

Figure 5. Differences in cytotoxic anti-neoplastic potency for gemcitabine-( $\mathrm{C}_{4}$-amide $)$-[anti-HER2/neu] compared to gemcitabine alone. Legends: (4) covalent gemcitabine- $\left(\mathrm{C}_{4^{-}}\right.$ amide)-[anti-HER2/neu] immunochemotherapeutic (182 hour incubation period); (•) gemcitabine chemotherapeutic (72hour incubation period); and (A) gemcitabine chemotherapeutic (182-hour incubation period). Chemotherapeuticresistant mammary adenocarcinoma (SKBr-3) monolayer populations were incubated with covalent gemcitabine $\left(\mathrm{C}_{4^{-}}\right.$ amide)-[anti-HER2/neu] or gemcitabine formulated in triplicate at gradient gemcitabine-equivalent concentrations. Cytotoxic anti-neoplastic potency was measured using a MTT cell vitality assay relative to matched negative reference controls. 
globulin fragments (e.g. Fab'), receptor ligands or other biologically active protein fractions can be achieved utilizing only a relatively small array of organic chemistry reaction schemes. Chemical sites within gemcitabine that are potentially available for synthetic covalent bond reactions include the $\left(\mathrm{C}_{4}{ }^{\prime}\right)-\mathrm{NH} 2,\left(\mathrm{C}_{3}{ }^{\prime}\right)-\mathrm{OH}$ and $\left(\mathrm{C}_{5}{ }^{\prime}\right)-\mathrm{OH}$ groups that can be reversibly protected utilizing di-tertdibutyl dicarbonate [61] when non-selective organic chemistry reaction schemes are employed. Generation of a covalent bond at the $\mathrm{C}_{5}$-methylhydroxy group of gemcitabine represents one molecular approach to synthesizing covalent gemcitabine-immunochemotherapeutics or gemcitabine-ligand preparations [36,40,56-61,76]. A second and possibly more infrequently utilized organic chemistry reaction involves the creation of a covalent bond at the cytosine-like $\mathrm{C}_{4}$-amine group of gemcitabine either in the form of a direct link to a "targeting" platform for selectivey chemotherapeutic delivery or alternatively for the purpose of creating a gemcitabine reactive intermediate $[21,59,61,77,78]$. Similar molecular strategies have been employed to synthesize covalent anthracycline immunochemotherapeutics through the formation of a covalent bond at the $\alpha$-monoamine $\left(\mathrm{C}_{3}\right.$-amine) group associated with the carbohydrate-like moiety of doxorubicin, daunorubicin, or epirubicin [5,7-9,11-16,18,19,23, $71,72]$. In addition to the anthracyclines [72] and gemcitabine analogous organic chemistry reaction schemes employing succinimidyl 4,4-azipentanoate could potentially be applied to covalently bond cytosine arabinoside (Ara-C), 5-azacytidine, cladribine (2-chloro2'deoxyadenine), clofarabine, decitabine (5-aza-2'deoxycytidine), fludarabine, lenalidamide, troxacitabine or other chemotherapeutic (pharmaceutical) agents that contain an available mono-amine group to large molecular weight platforms like monoclonal immunoglobulin.

Gemcitabine has been covalently bound to biologically relevant ligands that inludes poly-L-glutamic acid (polypeptide configuration), [58] cardiolipin, [56, 57] 1-dodecylthio-2-decyloxypropyl-3-phophatidic acid, [40,60] lipid-nucleosides, [76] $N$-(2-hydroxypropyl) methacrylamide polymer (HPMA), [21] benzodiazepine receptor ligand, [59,61] 4-(N)-valeroyl, 4-(N)-lauroyl, 4$(N)$-stearoyl, [78] 1,1',2-tris-noraqualenecarboxylic acid, [79] and the 4-fluoro [18F]-benzaldehyde derivative [77] for application as a positron-emitting radionuclide. Few if any published have described the molecular design, chemical synthesis and evaluation of the cytotoxic antineoplastic potency for gemcitabine immunochemotherapeutic created by generating a covalent bond at either the $\mathrm{C}_{5}$-methylhydroxy [36] or cytosine-like $\mathrm{C}_{4}$-amine groups of gemcitabine. In addition, there has to date been no previously published descriptions of utilizing succinimidyl 4,4-azipentanoate to create a UV-photoactivated gemcitabine- $\left(\mathrm{C}_{4}\right.$-amide $)$ intermediate to facilitate

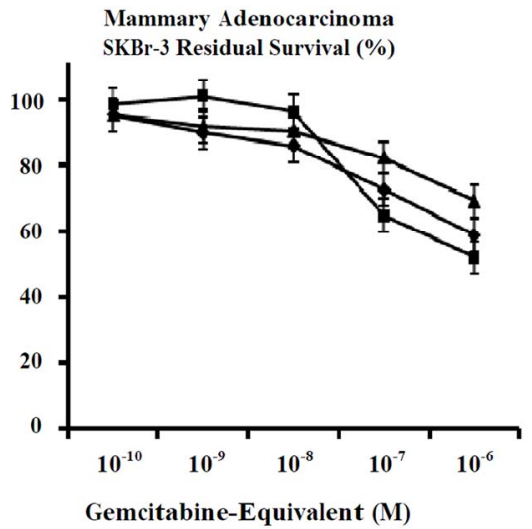

Figure 6 .Relative cytotoxic anti-neoplastic potency for gemcitabine-( $\mathrm{C}_{4}$-amide)-[anti-HER2/neu] and gemcitabine$\left(\mathrm{C}_{4}\right.$-amide)-[anti-HER2/neu] compared to gemcitabine alone. Legends: (4) covalent gemcitabine-( $\mathrm{C}_{4}$-amide)-[anti-HER2/ neu] immunochemotherapeutic (182-hours); (A) covalent gemcitabine-( $\mathrm{C}_{5}$-methylcarbamate)-[anti-HER2/neu] immunochemotherapeutic (182-hour incubation period) and (•) gemcitabine chemotherapeutic (72-hour incubation period) Chemotherapeutic-resistant mammary adenocarcinoma (SKBr-3) monolayer populations were incubated individually with gemcitabine-( $\mathrm{C}_{4}$-amide $)$-[anti-HER2/neu], gemcitabine $\left(\mathrm{C}_{5}\right.$-methylcarbamate)-[anti-HER2/neu] or gemcitabine formulated in triplicate at gradient gemcitabine-equivalent concentrations. Cytotoxic anti-neoplastic potency was measured using a MTT cell vitality assay relative to matched negative reference controls.

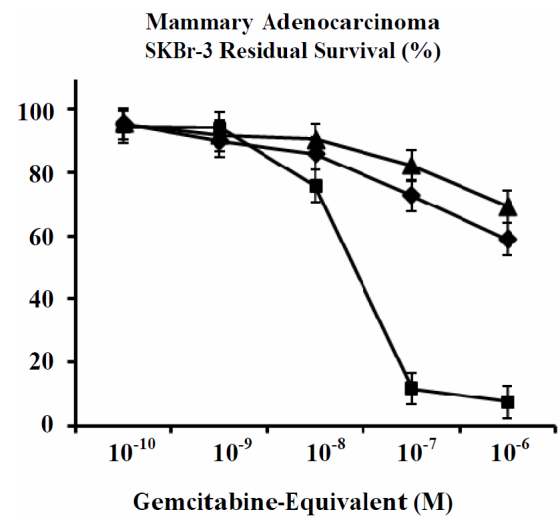

Figure 7. Relative cytotoxic anti-neoplastic potency of covalent gemcitabine-( $\mathrm{C}_{4}$-amide $)$-[anti-HER2/neu] and gemcitabine-( $\mathrm{C}_{5}$-methylcarbamate)-[anti-HER2/neu] immunochemotherapeutics as a function of gemcitabine-equivalent concentrations. Legends: (4) gemcitabine-( $\mathrm{C}_{4}$-amide)-[anti-HER2/ neu]; (A) gemcitabine-( $\mathrm{C}_{5}$-methyl carbonate)-[anti-HER2/neu]; and $(\bullet)$ gemcitabine alone. Chemotherapeutic-resistant mammary adenocarcinoma (SKBr-3) monolayer populations were incubated 182-hours with covalent gemcitabine immunochemotherapeutics or gemcitabine formulated in triplicate at gradient gemcitabine-equivalent concentrations. Cytotoxic anti-neoplastic potency was measured using a MTT cell vitality assay relative to matched negative reference controls. 


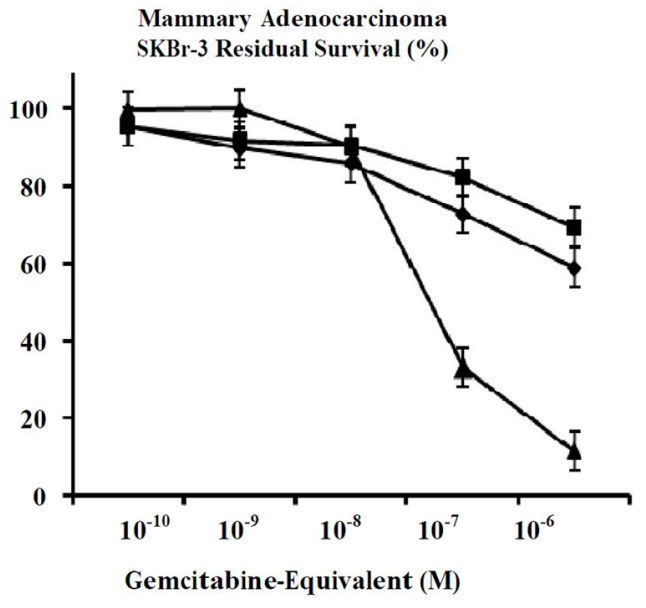

Figure 8. Relative cytotoxic anti-neoplastic potency of the covalent immunochemotherapeutics gemcitabine- $\left(\mathrm{C}_{4}\right.$-amide $)$ [anti-HER2/neu], gemcitabine-( $\mathrm{C}_{5}$-methyl-carbamate)-[anti-HER2/ neu] and epirubicin-(C3-amide)-[anti-HER2/neu] formulated at chemotherapeutic-equivalent concentrations. Legends: (4) gemcitabine-( $\mathrm{C}_{4}$-amide)-[anti-HER2/neu] at 182-hours; ( $\bullet$ gemcitabine-( $\mathrm{C}_{5}$-methylcarbonate)-[anti-HER2/ neu] at 182-hours; and (A) epirubicin-(C3-amide)-[anti-HER2/neu] at 72-hours. Chemotherapeutic-resistant mammary adenocarcinoma (SKBr3) monolayer populations were incubated with the covalent immunochemotherapeutics or gemcitabine chemotherapeutic that were each formulated in triplicate at gradient chemotherapeutic-equivalent concentrations. Cytotoxic anti-neoplastic potency was measured using a MTT cell vitality assay relative to matched negative reference controls.

synthesis of a covalent gemcitabine immunochemotherapeutic similar to gemcitabine-( $\mathrm{C}_{4}$-amide)-[anti-HER2/ neu] (Figure 1). Analogous synthetic organic chemistry reaction schemes have however been published on a very limited scale for the production of a covalent epirubicin- $\left(\mathrm{C}_{3}\right.$-amide $)$-[anti-HER2/neu $]$ immunochemotherapeutic [72].

Speicific attributes related to the variables of 1) chemotherapeutic chemical composition; 2) organic chemistry reaction selectivity; 3) molar ratio formulations (chemotherapeutic/reagent/IgG); 4) specific sequential order of individual organic chemistry reaction schemes, and 5) extension of incubation periods for organic chemistry reactions during the synthesis of gemcitabine ( $\mathrm{C}_{4}$-amide)-[anti-HER2/neu], gemcitabine- $\left(\mathrm{C}_{5}\right.$-methylcarbamate)-[anti-HER2/neu] and epirubicin-( $\mathrm{C}_{3}$-amide)[anti-HER $2 / n e u$ ] collectively minimized side reactions resulting in the formation of extraneous side-products (Figure 1) [36,72]. Reaction condition variables are especially important during the initial phases of synthesizing gemcitabine- $\left(\mathrm{C}_{5}\right.$-methylcarbamate $)$ and UV-photo-activated gemcitabine- $\left(\mathrm{C}_{4}\right.$-amide $)$ reactive intermediates (Figure 2). [36] Generation of the UV-photoactivated gemcit- abine-( $\mathrm{C}_{4}$-amide $)$ intermediate with succinimidyl 4,4-azipentanoate involves the succinimide ester group

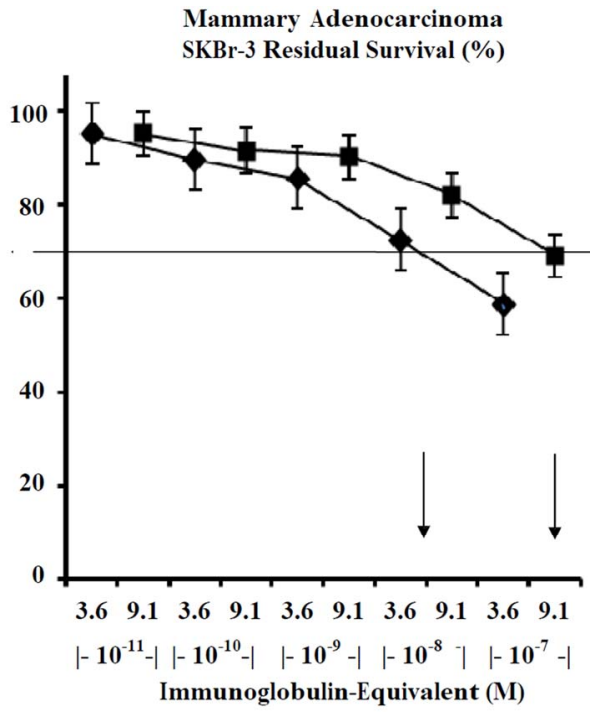

Figure 9. Relative cytotoxic anti-neoplastic potency of gemcitabine-( $\mathrm{C}_{4}$-amide $)$-[anti-HER2/neu] and gemcitabine- $\left(\mathrm{C}_{5}\right.$-methylcarbamate)-[anti-HER2/neu] as a function of immunoglobulin-equivalent concentration. Legends: (4) gemcitabine-( $\mathrm{C}_{4}$-amide $)$-[anti-HER2/neu] with a gemcitabine molar-incorporation-index of 2.78:1 (182-hour incubation period); and (•) gemcitabine-( $\mathrm{C}_{5}$-methyl carbonate $)$-[anti-HER2/ $n e u$ ] with a gemcitabine molar-incorporation-index of 1.1:1 (182-hour incubation period). Arrows indicate the approximate concentration of gemcitabine-( $\mathrm{C}_{4}$-amide $)$ [anti-HER2/neu] and gemcitabine- $\left(\mathrm{C}_{5}\right.$-methyl carbonate $)$ [anti-HER2/neu] necessary to achieve a $\mathbf{3 0} \%$ level of cytotoxic anti-neoplastic potentcy. Chemotherapeutic-resistant mammary adenocarcinoma (SKBr-3) monolayer populations were incubated with either covalent gemcitabine immunochemotherapeutics formulated in triplicate at gradient concentrations. $\mathrm{Cy}$ totoxic anti-neoplastic potency measured using a MTT cell vitality assay relative to matched negative reference controls.

preferentially reacting with and forming a colvaent bond at the $\mathrm{C}_{4}$ cytosine-like amine group of gemcitabine. In organic solvent systems like DMSO and DMF succinimidyl 4,4-azipentanoate may also react to a much lesser degree with nitrogen groups embended within five or six member ring structures but such complexes reportedly dissociate redily with the addition of small amounts of $\mathrm{ddH}_{2} \mathrm{O}$ or aqeous buffer An organic solvent in the form of DMSO was applied in these investigations in order to preserve the integrity of the UV-photoactivated moiety of succinimidyl 4,4-azipentanoate during the extended incubation with gemcitabine. Alternatively, an aqueous buffer formulated between the $\mathrm{pH}$ range of 7 to 9 can effectively promote covalent amide bond formation when shorter incubation periods are indicated. Utilization of aqueous buffer with a $\mathrm{pH}$ of 6.5 and implementation of lower reaction condition temperatures $\left(\right.$ e.g. $\left.4^{\circ} \mathrm{C}\right)$ have reportedly been found to enhance the reaction of succinimide ester group with dif 


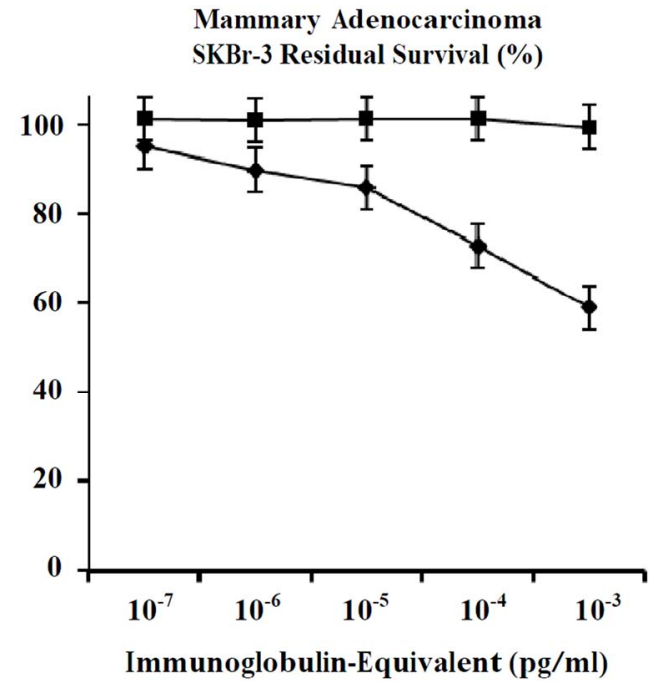

Figure 10. Relative cytotoxic anti-neoplastic potency of covalent gemcitabine-( $\mathrm{C}_{4}$-amide $)$-[anti-HER2/neu] immunochemotherapeutic compared to anti-HER2/neu monoclonal immunoglobulin. Legends: (4) covalent gemcitabine- $\left(\mathrm{C}_{4^{-}}\right.$ amide)-[anti-HER2/neu] immunochemotherapeutic; and (A) anti-HER2/neu monoclonal immunoglobulin. Chemotherapeutic-resistant mammary adenocarcinoma (SKBr-3) monolayer populations were incubated with gemcitabine-( $\mathrm{C}_{4^{-}}$ amide)-[anti-HER2/neu] and anti-HER2/neu monoclonal immunoglobulin formulated in triplicate at at gradient concentrations. Cytotoxic anti-neoplastic potency was measured using a MTT cell vitality assay relative to matched negative reference controls.

ferent primary amine subtypes (e.g. lysine $\varepsilon$-aminevspeptide $N$-terminal amine).

Conservative speculation suggests that one of the reasons for the differences in molar incorporation indexes (2.78-vs-1.1) for gemcitabine-( $\mathrm{C}_{4}$-amide)-[anti-HER2 Ineu compared to gemcitabine-( $\mathrm{C}_{5}$-methylcarbamate $)$ [anti-HER2/neu] respectively was probably due to a combination of two critical reaction condition variables. Most notable in this regard was the application of the UV-photoactivated gemcitabine-( $\mathrm{C}_{4}$-amide $)$ intermediate at a 10-to-1 molar ratio to anti-HER2/neu monoclonal immunoglobulin in concert with a lack of a requirement [72] for 2-iminothiolane (2-IT) [36,71] or $N$-succinimidyl-S-acetylthioacetate (SATA) [7] to pre-thiolate immunoglobulin fractions, IgG fragments, receptor ligands or other biologically active peptide proteins (Figure 1). Higher molar incorporation indexes are possible to achieve with certain methodology modifications but the harsher synthesis conditions required for such purposes almost invariably are accompanied by substantial reductions in final product yield of the covalent immunochemotherapeutic [6]. In addition to harsh reaction conditions, immunoglobulin antigen binding-avidity can be reduced as a function of excessive covalent chemotherapeutic incorporation into or within the Fab antigen- binding domain of immunoglobulin fractions. Despite this consideration, relatively higher molar incorporation indexes were attained during the synthesis of gemcitabine-( $\mathrm{C}_{4}$-amide)-[anti-HER2/neu] (2.78-to-1 or $278 \%)$ compared to gemcitabine-( $\mathrm{C}_{5}$-methylcarbamate $)$ [anti-HER2/neu] (1.1-to-1 or 110\%), [36] epirubicin(C13-imino)-[anti-HER2/neu] (0.4-to-1 or $40 \%)$, [71] epirubicin-(C3-amide)-[anti-HER2/neu] (0.275 - to- 1 or $27.5 \%),[7,72]$ and epirubicin-(C3-amide)-[anti-EGFR] (0.407-to-1 or 40.7\%) [7]. Conservative speculation suggests that one reason for the higher molar incorporation index observed for covalent gemcitabine-( $\mathrm{C}_{4}$-amide $)$ [anti-HER2/neu] immunochemotherapeutic was due to the implementation of a synthesis scheme that involved a distinctly different organic chemistry reactions and that even higher molar incorporation indexes along with greater levels of cytotoxic anti-neoplastic potency are possible (Figure 1).

A somewhat unique property of the UV-photoactivated gemcitabine- $\left(\mathrm{C}_{4}\right.$-amide $)$ intermediate generated utilizing succinimidyl 4,4-azipentanoate in Phase-I of the synthetic organic chemistry reaction scheme is that it does not contain a sulfhydryl-reactive maleimide group (Figure 1). The lack of a sulfhydryl-reactive maleimide moiety within the structure of the UV-photoactivated gemcitabine-( $\mathrm{C}_{4}$-amide $)$ intermediate therefore allows it to be applied to synthesize covalent immunochemotherapeutics without a requirement to pre-thiolateamine groups associated with lysine residues in the amino acid sequence of anti-HER2/neu monoclonal immunoglobulin. Because of this feature it is possible to initiate Phase-II of the synthetic organic chemistry reaction scheme for gemcitabine- $\left(\mathrm{C}_{4}\right.$-amide $)$-[anti-HER2/neu] without the introduction of reduced sulfhydryl groups into the amino acid sequence of immunoglobulin ( $\operatorname{IgG}$ ) fractions, IgG fragments $\left[\mathrm{F}\left(\mathrm{ab}^{\prime}\right)_{2}\right.$ or $\left.\mathrm{Fab}^{\prime}\right]$, receptor ligands, receptor ligand fragments or other biologically relevant protein fractions (Figure 1). In contrast, the gemcitabine$\left(\mathrm{C}_{5}\right.$-methylcarbamate $)$ reactive intermediate synthesized with $N$-[ $p$-maleimidophenyl]-isocyanate does contain a sulfhydryl-reactive maleimide group (Figure 2) [36]. Similarly, anthracycline reactive intermediates applied to synthesize many if not most anthracycline-immunochemotherapeutics also employ a sulfhydryl-reactive maleimide group to facilitate the creation of a covalent bond with immunoglobulin or other biologically active protein fractions $[7,71,72]$. Such synthetic organic chemistry reactions schemes are dependent upon the utilization of heterobifunctional reactants similar to succinimidyl-4$(\mathrm{N}$-maleimidomethyl) cyclohexane-1-carboxylate (SMCC), [7,80-82] N- $\varepsilon$-maleimidocaproic acid hydrazide (EMCH), $[9,10,71]$ or $N$-[ $p$-maleimidophenyl]- isocyanate (PMPI) [36,53-55]. In the application of these covalent bondforming reagents, disruption of disulfide bond structures 
or prethiolation of immunoglobulin or other biological protein fractions is almost invariably required due to the relatively low number of non-sterically hindered sulfhydryl groups available within the amino acid sequence of most biologically active proteins in the form of reduced cysteine amino acid residues (e.g. R-SH). Increasing the number of available reduced sulf- hydryl groups can be achieved by the application of 1,4dithiothreitol which reduces intramolecular cystinecystine [26-28] and similar disulfide structures [83] (DTT: R-CH2-S-S-CH2-R-2 $\mathrm{R}-\mathrm{CH} 2-\mathrm{SH}$ ). The actual synthetic introduction of "new" or additional reduced sulfhydryl groups at the $\varepsilon$-amine of lysine amino acid residues is possible utilizing organic chemistry reaction schemes that employ 2-iminothiolane (2-IT), [2,6, 36,71,84] mercaptosuccinimide, [85] or $\mathrm{N}$-succinimidyl $S$-acetylthioacetate (SATA) $[7,84,86]$. Alternatively, carboxyl groups on molecules like heparin and hyaluronic acid (HA) can be thiolated with 3,3' dithiobis (propanoic)hydrazide (DPTH) [83,87] or divinylsulfone (DVS), [88, 89] in addition to the hydroxyl groups of molecules with a cholesterol-like core [90]. In the application of DTPH the integral disulfide bond is subsequently reduced with DTT reagent [83,87].

Covalently bonding gemcitabine or other chemotherapeutic agents to biological protein fractions like immunoglobulin without a requirement to convert existing cystine-cystine disulfide bonds to their reduced form (R1-S-S-R2-R1-SH and R2-SH) or the synthetic introduction of reduced sulfhydryl groups provides several disctinct advantages. Specifically, such synthetic organic chemistry reaction schemes entail the implementation of fewer synthetic chemistry reactions, require fewer critical reagents, and maximize final "end-product" yield due in part to at least one less column chromatography separation procedure. The brief duration of the synthetic organic chemistry reaction scheme for gemcitabine$\left(\mathrm{C}_{4}\right.$-amide)-[anti-HER2/neu] utilizing succinimidyl 4,4azipentanoate is realized because of the relatively rapid time course for the Phase-I, but especially the Phase-II organic chemistry reaction. The synthetic organic chemistry reaction scheme has also been designed so that adjustment of buffer $\mathrm{pH}$ to different levels during the procedure is not necessary in contrast to other techniques [91]. Perhaps one of the most important features of the synthesis methodology is a lack of a requirement for cystinecystine disulfide bond reduction or pre-thiolation that in turn allows by design the application of synthetic chemistry reactions that are highly efficient under relatively mild conditions thereby possing a lower risk of protein fragmentation or polymerization (e.g. $\mathrm{IgG}-\mathrm{IgG}$ ) through premature intra-molecular disulfide bond formation [2]. Realized benefits therefore include greater retained biological activity (e.g. antigen binding-avidity) and increased total final yield of a function immuno chemotherapeutic end-product. Lastly, lack of a requirement to either convert existing cystine-cystine disulfide bonds to their reduced form or the introduction of reduced sulfhydryl groups into immunoglobulin fractions reduces restrictions and limitations on the magnitude of the molar-incorporation-index that can be attained. In contrast, the chemotherapeutic incorporation index for covalent immunochemotherapeutics synthesized utilizing SMCC, [7,80-82] EMCH [9,10,71] or PMPI [36,53-55] is only equivalent to or lower than the extent of prethiolation at $\varepsilon$-amine groups associated with the finite number of lysine residues within the amino acid sequence of protein fractions. In prethiolation dependent synthesis schemes higher epirubicin molar-incorporationindexes are possible with modifications in methodology but requires the use of harsher synthesis conditions that are frequently accompanied by substantial reductions in total yield of covalent immunochemotherapeutic, [6] and declines in antigen-immubnoglobulin bindingavidity (e.g. cell-ELISA parameters). Presumably the 7.6 fold higher potency of gemcitabine-( $\mathrm{C}_{4}$-amide $)$-[anti-HER2/neu] compared to gemcitabine- $\left(\mathrm{C}_{5}\right.$-methylcarbamate $)$-[anti-HER2/ $n e u]$ at the cytotoxic anti-neoplastic potency level of approximately $30 \%$ can be attributed to a combination of a greater degree retained biological activity for antiHER2/neu (cell-ELISA) and a higher gemcitibin molar-incorporation-index of 2.78-to-1 for gemcitabine- $\left(\mathrm{C}_{4^{-}}\right.$ amide)-[anti-HER2/neu] in contrast to 1.1-to-1 for gemcitabine-( $\mathrm{C}_{5}$-methylcarbamate)-[anti-HER2/neu] (Figure 9). Both of these properties are anticipated to be attributable to the application of gentler reaction conditions in part due to a lack of a requirement for anti-HER $2 / n e u$ prethiolation during Phase-II synthesis reaction sheme for gemcitabine-( $\mathrm{C}_{4}$-amide $)$-[anti- HER2/neu].

Implementation of succinimidyl 4,4-azipentanoate in the synthesis scheme for gemcitabine- $\left(\mathrm{C}_{4}\right.$-amide $)$-[antiHER $1 / n e u$ ] offers desirable attributes other than a lack of a requirement for pre-thiolation of immu- noglobulin or similar molecular platforms that possess biological activity that affords properties of selective "targeted" delivery. In contrast to SMCC, [7,80-82] EMCH $[9,10,71]$ or PMPI [36,53-55] the synthesis of gemcitabine- $\left(\mathrm{C}_{4}\right.$-amide)-[anti-HER2/neu] utilizing succinimidyl 4,4-azipentanoate has the added benefit of not introducing extraneous five and six carbon or carbon/nitrogen ring structures into the final covalent immunochemotherapeutic endproduct (Figures 1 and 2). Elimination of extraneous ring structures decreases the probability of inducing in-vivo humoral immune response when administered by IV injection that can ultimately result in the formation of neutralizing antibody titers and an increased risk of post-treatment immune hypersensitivity reactions. In addition, the Phase-I synthetic organic chemistry reaction scheme can be performed in either aqueous buffer, or 
Cytotoxic Anti-Neoplastic Activity against Chemotherapeutic-Resistant Mammary Adenocarcinoma SKBr-3

organic solvent systems supplemented with triethylamine $\left[\mathrm{N}\left(\mathrm{CH}_{2} \mathrm{CH}_{3}\right)_{3}\right]$ or similar proton acceptor molecules at low concentrations. In stock solutions of reaction mixtures formulated in aqueous buffers a significant amount of hydrolytic degradation of succinimidyl 4,4-azipentanoate is expected to occur to varying degrees. Alternatively, if stock solutions and reaction mixtures of gemcitabine and succinimidyl 4,4-azipentanoate are instead formulated in an anhydrous organic solvent like DMSO in combination with a proton acceptor molecule then the resulting UV-photoactivated gemcitabine- $\left(\mathrm{C}_{4}\right.$-amide $)$ intermediate is stable at $4{ }^{\circ} \mathrm{C}$ or $-20^{\circ} \mathrm{C}$ for a period of time when adequately protected from UV-light exposure. Such chemical properties of succinimidyl 4,4-azipentanoate allow for the convenient option of "presynthesizing" and preserved storage of the UV-photoactivated gemcitabine- $\left(\mathrm{C}_{4}\right.$-amide $)$ intermediate for an extend period of time for the future production of covalent gemcitabine-immunochemotherapeutics [72]. The synthetic organic chemistry reaction scheme described also offers another added level of convenience because it represents a template model that can be adapted and modified to facilitate the covalent bonding of an array of different chemotherapeutic agents to a wide range of molecular platforms that can facilitate selective "targeted" pharmaceutical delivery.

Cell-Binding Profiles-Increases in standardized immunoglobulin-equivalent concentrations of gemcitabine$\left(\mathrm{C}_{4}\right.$-amide)-[anti-HER2/neu] and gemcitabine-( $\mathrm{C}_{5}$-methylcarbamate)-[anti-HER2/neu] correlated with elevations in total immunoglobulin membrane binding profiles in populations of human mammary adenocarcinoma detected by cell-ELISA (Figure 4). The lower standardized immunoglobulin-equivalent concentration range for gemcitabine-( $\mathrm{C}_{4}$-amide $)$-[anti-HER2/neu] compared to gemcitabine-( $\mathrm{C}_{5}$-methylcarbamate)-[anti-HER2/neu] implies that the former covalent gemcitabine immunochemotherapeutic may have a higher level of retained antiHER2/neu binding-avidity. The most probably explanation for this difference can be attributed to the implementation of milder organic chemical reaction conditions and a lack of a requirement for pre-thiolate of antiHER2/neu fractions. Previous investigations have similarly noted that modest alterations in synthetic chemistry and elevations in the chemotherapeutic molar incorporation index can profoundly influence immunoglobulin binding properties [29].

Cytotoxic Anti-Neoplastic Activity/Potency-Covalent gemcitabine conjugates have been synthesized that exert greater cytotoxic anti-neoplastic potency than gemcitabine chemotherapeutic alone, but these preparations have been produced in the form of gemcitabine(oxyether phopholipid) [40,60] or dual gemcitabine/
doxorubicin-HPMA (N-(2-hydroxypropyl) methacrylamide polymer). [21] In a very limited number of investigations, the cytotoxic anti-neoplastic activity for majorities of these covalently bonded gemcitabine preparations were reported against human mammary carcinoma (MCF7/WT-2'), [60] human mammary adenocarcinoma (BG-1), [60] promyelocytic leukemia, [40,60] a T-4 lymphoblastoid clone, [60] glioblastoma, [40,60] cervical epithelioid carcinoma, [60] colon adenocarcinoma, [60] pancreatic adenocarcinoma, [60] pulmonary adenocarcinoma, [60] oral squamous cell carcinoma, [60] and prostatic carcinoma [21].

Increases in the molar chemotherapeutic-equivalent concentrations of gemcitabine-( $\mathrm{C}_{4}$-amide)-[anti-HER2/neu] and gemcitabine-( $\mathrm{C}_{5}$-methylcarbamate)-[anti-HER2/ neu] created corresponding elevations in the cytotoxic antineoplastic potency and declines in the residual survival of chemotherapeutic-resistant mammary adenocarcinoma (SKBr-3) populations (Figures 5-7). Neither gemcitabine- $\left(\mathrm{C}_{4}\right.$-amide $)$-[anti-HER2/neu] or gemcitabine- $\left(\mathrm{C}_{5}\right.$-methylcarbamate)-[anti-HER2/neu] exerted substantially greater selective "targeted" anti-neoplastic potency against chemotherapeutic-resistant mammary adenocarcinoma ( $\mathrm{SKBr}-3)$ that was greater than gemcitabine alone when formulated at molar chemotherapeutic-equivalent concentrations between $10^{-10} \mathrm{M}$ to $10^{-6} \mathrm{M}$ and an incubation period of 182-hours (Figures 5-7). Such findings are in contrast to covalent epirubicin-[anti-HER2/neu] immunochemotherapeutics that possess equivalent or greater cytotoxic anti-neoplastic potency levels than epirubicin alone $[7,71,72]$. Despite this difference, the selectively "targeted" cytotoxic anti-neoplastic potency of covalent gemcitabine-( $\mathrm{C}_{4}$-amide)-[anti-HER2/neu] and gemcitabine- $\left(\mathrm{C}_{5}\right.$-methylcarbamate $)$-[anti-HER2/neu] immunochemotherapeutics at 182-hours was almost identicial to levels exerted by gemcitabine after a 72-hour incubation period [92].

In the interpretation of the cytotoxic anti-neoplastic potency of gemcitabine-( $\mathrm{C}_{4}$-amide $)$-[anti-HER2/neu] and gemcitabine-( $\mathrm{C}_{5}$-methylcarbamate)-[anti-HER2/neu] it should be emphasized that such comparisons were made at gemcitabine-equivalent concentrations. Alternatively, if comparisons of cytotoxic anti-neoplastic potency for the two covalent gemcitabine-immunochemotherapeutics are made as a function of immunoglobulin-equivalent concentrations (e.g. anti-HER2/neu content) and gemcitabine molar-incorporation-indexes then it is possible to detect a relatively greater level of potency for gemcitabine$\left(\mathrm{C}_{4}\right.$-amide)-[anti-HER2/neu] compared to gemcitabine $\left(\mathrm{C}_{5^{-}}\right.$ methylcarbamate)-[anti-HER2/neu] (Figure 9). Given this perspective, gemcitabine-( $\mathrm{C}_{4}$-amide)-[anti-HER2/neu] and gemcitabine-( $\mathrm{C}_{5}$-methylcarbamate)-[anti- HER2/neu] for example each exerted a $30 \%$ level of cytotoxic anti-neo- 
plastic potency at immunoglobulin-equivalent concentrations of $6.9 \times 10^{-8} \mathrm{M}$ and $9.1 \times 10^{-9} \mathrm{M}$ respectively (Figure 9). Based on these calculations, gemcitabine( $\mathrm{C}_{4}$-amide)-[anti-HER2/neu] was approximately 7.6 -fold more potent than gemcitabine- $\left(\mathrm{C}_{5}\right.$-methylcarbamate)[anti-HER2/neu] when cytotoxic anti-neoplastic activity was standardized as a function of immunoglobulinequivalent concentration. Presumably this difference in cytotoxic anti-neoplastic potency was due to a combination of a greater degree of retained biological activity for anti-HER2/neu (cell-ELISA) and a higher gemcitibin molar-incorporation-index (2.78-to-1) for gemcitabine$\left(\mathrm{C}_{4}\right.$-amide)-[anti-HER2/neu] compared to gemcitabine$\left(\mathrm{C}_{5}\right.$-methylcarbamate)-[anti-HER2/neu]. Both of these properties are likely attributable to the application of gentler reaction conditions again due in part to a lack of a requirement for anti-HER2/neu prethiolation during Phase-II of the organic chemistryreaction scheme applied in the synthesis of gemcitabine- $\left(\mathrm{C}_{4}\right.$-amide)-[anti-HER2/ neu].

In contrast to most covalent anthracycline immunochemotherapeutics described to date, a longer 182-hour incubation period was applied to access the cytotoxic anti-neoplastic potency of gemcitabine- $\left(\mathrm{C}_{4}\right.$-amide $)$-[antiHER2/neu ] and gemcitabine-( $\mathrm{C}_{5}$-methylcarbamate)-[antiHER2/neu] in order to optimally evaluate their cytotoxic anti-neoplastic potency (Figure 5) [36,71,72]. Longer incubation periods have also been applied to evaluate other synthetic gemcitabine-ligand preparations in order to more accurately access their ex-vivo cytotoxic anti-neoplastic potency $[21,36,40,59]$. Several explanations may account for the requirement to use longer incubation periods for the ex-vivo evalution of gemcitabine compared to anthracycline-immunochemotherapeutics or anthracycline covalent bound to other molecular platforms with properties that afford selective "targeted" delivery (e.g. receptor ligands). Since the covalent immunochemotherapeutics gemcitabine- $\left(\mathrm{C}_{4}\right.$-amide $)$-[antiHER2/neu], gemcitabine-( $\mathrm{C}_{5}$-methylcarbamate)-[anti-HER2/ $n e u$ ] [36] and several epirubicin-[anti-HER2/ neu] immunochemotherapeutics $[7,71,72]$ all selectively "target" chemotherapeutic delivery at the same HER2/ neu receptor site highly over-expressed on the external surface membrane of mammary adenocarcinoma (SKBr-3), it is possible that differences in their cytotoxic anti- neoplastic activity may be attributable to, 1) differences in the vulnerability of covalent bond structures created during the synthesis of gemcitabine-( $\mathrm{C}_{4}$-amide $)$-[anti-HER2/neu] and gemcitabine-( $\mathrm{C}_{5}$-methylcarbamate)-[anti-HER2/neu] to enzyme-mediated degradation or simple hydrolysis within the acidic endosome/lysosome microenvironment; 2) variations in the expression profile for different enzyme fractions necessary for biochemically liberating gemcitabine versus epirubicin from covalent immunochemotherapeutics; 3 ) variation in the acidic characteristics associated with the endosome/lysosome microenvironment necessary for liberating gemcitabine versus epirubicin from covalent immunochemotherapeutics; 4) greater capacity of the anthracycline moiety within intact covalent epirubicin immunochemotherapeutics to exert one or more of the multiple mechanisms-of-action recognized for this class of chemotherapeutic agent; 5) vulnerability of the gemcitabine moiety in covalent gemcitabine immunochemotherapeutics to inactivation by deamination. The fact that cytotoxic anti-neoplastic potency profiles for gemcitabine-( $\mathrm{C}_{4}$-amide)-[anti-HER2/ $n e u]$ and gemcitabine-( $\mathrm{C}_{5}$-methylcarbamate)-[anti-HER2/ $n e u]$ at the end of a 182-hour incubation period were very similar to those for gemcitabine after a 72-hour incubation period implies that cytotoxic anti-neoplastic activity of the gemcitabine immunochemotherapeutics is possibly delayed due to a slow release of the chemotherapeutic moiety that is apparently longer compared to the rate of anthracycline-release from covalent epirubicin-immunochemotherapeutics [7,71,72]. One important implication of this possible explanation is that a delayed and prolonged release or liberation of gemcitabine from covalent gemcitabine-( $\mathrm{C}_{4}$-amide $)$-[anti-HER2/neu] and gemcitabine-( $\mathrm{C}_{5}$-methylcarbamate)-[anti-HER2/neu] could represent a desirable property that can be employed as a molecular strategy to evoke "super-loading" that in turn can facilitate extensive and sustained chemotherapeutic deposition and release within populations of neoplastic cells.

Collective interpretation of results from SDS-PAGE/ immunodection/chemiluminscent autoradiography, cellELISA and cytotoxic anti-neoplastic potency analyses illustrates how gemcitabine can be covalently bound to a large molecular weight "carrier" (protein) to facilitate selective "targeted" chemotherapeutic delivery and cytotoxic anti-neoplastic potency. The positive findings directly address one of the major objectives that originally motivated the molecular design and synthesis of gemcitabine-( $\mathrm{C}_{4}$-amide)-[anti-HER2/neu]. Additionally, there was a perceived need for the molecular design of a synthesis scheme that was composed of a sequential series of organic chemistry reactions that could facilitate relatively rapid production of gemcitabine-( $\mathrm{C}_{4}$-amide $)$-[anti-HER2/ $n e u]$ using mild conditions that affored minimal degradative low molecular weight fragmentation or large molecular weight polymerization (e.g. IgG-IgG). Recent investigations describing the methodology employed for the synthesis of epirubicin-( $\mathrm{C}_{5}$-amide $)$-[anti-HER2/neu] through the application of a UV-photoactivated epirubicin intermediate revealed that there was a high degree of probability that a similar organic chemistry regimen 
Cytotoxic Anti-Neoplastic Activity against Chemotherapeutic-Resistant Mammary Adenocarcinoma SKBr-3

could be adapted as a model with minor modifications for the relatively rapid synthesis of a covalent gemcitabine-( $\mathrm{C}_{4}$-amide)-[anti-HER2/neu] immunochemotherapeutic [72]. In this context, a set of organic chemistry reactions were implemented to synthesize gemcitabine( $\mathrm{C}_{4}$-amide)-[anti-HER2/neu] that had not previously been described for the production of a gemcitabine-immunochemotherapeutic or covalent gemcitabine-ligand preparation. The organic chemistry synthesis reactions utilized for the production of gemcitabine- $\left(\mathrm{C}_{4}\right.$-amide $)$-[anti-HER2/ $n e u$ ] also possesses practical utility because it can serve as a model or template for the molecular design and production of other covalent immunochemotherapeutics.

Conceptually there are at least five analytical variables that could have alternatively been modified to achieve substantially higher total levels of cytotoxic anti-neoplastic potency for gemcitabine-( $\mathrm{C}_{4}$-amide)-[antiHER2/neu]. First, incubation times with chemotherapeutic-resistant mammary adenocarcinoma ( $\mathrm{SKBr}-3)$ could have been lengthened to a period $>182$-hours [36] there-by allowing greater opportunity for larger amounts of gemcitabine to be internalized by receptor-mediated endocytosis and subsequently liberated intracellularly from gemcitabine- $\left(\mathrm{C}_{4}\right.$-amide)-[anti-HER2/neu] or gemcitabine- $\left(\mathrm{C}_{5}\right.$-methylcarbamate $)$-[anti-HER2/neu]. Support for this consideration in based on the observation that there was a simple dose effect for gemcitabine- $\left(\mathrm{C}_{4}\right.$ amide)-[anti-HER2/neu], and because mammary adenocarcinoma (SKBr-3) survivability was very similar when challenged with gemcibatine-( $\mathrm{C}_{5}$-methylcarbamate)-[antiHER2/neu] (182-hours) compared to gemcitabine (72hours), but increased dramatically for gemcitabine when the incubation period was extended to 182-hours (Figures 5-7).[36] Conservative speculation suggests that incubation of chemotherapeutic-resistant mammary adenocarcinoma ( $\mathrm{SKBr}-3)$ with gemcitabine-( $\mathrm{C}_{4}$-amide $)$ [anti-HER2/neu] or gemcibatine-( $\mathrm{C}_{5}$-methylcarbamate)[anti-HER2/neu] for periods greater than 182-hours would have resulted in even higher levels of cytotoxic anti-neoplatic potency since there was no indication that the level of cytotoxic activity achieved against chemotherapeutic-resistant mammary adenocarcinoma (SKBr-3) had reached a "plateau" or maximum level (Figures 5-7).

Second, cytotoxic anti-neoplastic potency of gemcibatine- $\left(\mathrm{C}_{4}\right.$-amide $)$-[anti-HER2/neu $]$ and gemcitabine- $\left(\mathrm{C}_{5^{-}}\right.$ methylcarbamate)-[anti-HER2/neu] could have alternatively been assessed against a human neoplastic cell type that was not chemotherapeutic-resistant similar to cancer cell types utilized to evaluate majority of the covalent biochemotherapeutics reported in the literature to date. Rare exceptions to this consideration have been the application of chemotherapeutic-resistant metastatic melanoma M21 (covalent daunorubicin immunochemothera- peutics synthesized using anti-chondroitin sulfate proteoglycan 9.2.27 surface marker), [29,32,93] chemotherapeutic-resistant mammary carcinoma MCF-7AdrR (covalent anthracycline-ligand chemotherapeutics synthesized utilizing epidermal growth factor/EGF or an EGF fragment); [94] and chemotherapeutic-resistant mammary adenocarcinoma (SKBr-3) populations (epirubicin-anti-HER2/neu, epirubicin-anti-EGFR, gemcitabineHER2/neu) [7, 36,71,72].

Somewhat analogous to the concept of non-chemotherapeutic resistant cancer cell types, the cytotoxic antineoplatic potency of gemcibatine-( $\mathrm{C}_{4}$-amide $)$-[anti-HER2/ $n e u$ ] and gemcitabine-( $\mathrm{C}_{5}$ methylcarbamate)-[anti-HER2/ $n e u$ ] could also have alternatively been measured against an entirely different neoplastic cell type such as pancreatic carcinoma, [95] small-cell lung carcinoma, [96] neuroblastoma, [97] or leukemia/lymphoid [60,98] populations due to their relatively higher gemcitabine sensitivity. Similarly, human promyelocytic leukemia, $[40,60]$ T-4 lymphoblastoid clones, [60] glioblastoma, [40,60] cervical epitheliod carcinoma, [60] colon adenocarcinoma, [60] pancreatic adenocarcinoma, [60] pulmonary adenocarcinoma, [60] oral squamous cell carcinoma, [60] and prostatic carcinoma [21] have all been found to be sensitive to gemcitabine and gemcitabine-(oxyether phopholipid) covalently bonded chemotherapeutics. Within this array of neoplastic cell types, however, human mammary carcinoma (MCF-7/WT-2') [60] and mammary adenocarcinoma (BG-1) [60] are known to be relatively more resistant to gemcitabine and gemcitabine-(oxyether phopholipid) chemotherapeutic conjugate. Presumably this pattern of diminished gemcitabine sensitivity is directly relevant to the cytotoxic anti-neoplatic potency detected for gemcibatine-( $\mathrm{C}_{4}$-amide $)$ [anti-HER2/neu] and gemcitabine- $\left(\mathrm{C}_{5}\right.$-methylcarbamate $)$ -[anti-HER2/neu] compared to gemcitabine in chemotherapeutic-resistant mammary adenocarcinoma (SKBr-3) populations (Figures 5-7).

Third, cytotoxic anti-neoplastic potency of gemcibatine- $\left(\mathrm{C}_{4}\right.$-amide $)$-[anti-HER2/neu $]$ and gemcitabine- $\left(\mathrm{C}_{5^{-}}\right.$ methylcarbamate)-[anti-HER2/neu] could have been evaluated at higher gemcitabine-equivalent concentrations. Since gemcitabine in contrast to the anthracyclines has rarely been synthetically incorporated into (covalently bonded to) selective "targeted" delivery platforms, $[21,36,40,57-60]$ it is uncertain if this chemotherapeutic can be utilized to consistently create covalent gemcitabine immunochemotherapeutics that posses significantly higher levels of cytotoxic anti-neoplastic potency than gemcitabine alone (Figures 5-7) [36]. Despite this consideration, the paramount objective that moti- vates the molecular design and synthesis of covalent gemcitabine immunochemotherapeutics is the opportu- nity to 
create a new anti-cancer modality that affords reduced exposure of healthy tissues and organ systems to the cytotoxic anti-neoplastic properties of chemothera- peutics. By design, such attributes are facilitated by selectively "targeted" delivery of chemotherapeutic moieties in a manner that produces cytotoxic anti-neoplastic properties that are largely restricted to malignant lesions. Given this perspective and applying basic pharmacology principals, the variable of potency can simply be addressed through adjustment of concentration (dose administered) within the limitations of induced side effects and sequelae.

Fourth, anti-neoplastic potency of gemcitabine- $\left(\mathrm{C}_{4}-\right.$ amide)-[anti-HER2/neu] and gemcitabine- $\left(\mathrm{C}_{5}\right.$-methylcarbamate)-[anti-HER2/neu] would likely have been substantially greater if cellular proliferation had been assessed with either $\left[{ }^{3} \mathrm{H}\right]$-thymidine, or an ATP-based assay method because of their reportedly $\geq 10$-fold greater sensitivity in detecting early cell injury compared to MTT vitality stain based assay methods $[99,100]$. Despite this consideration, MTT vitality stain based assays continue to be extensively applied for the routine assessment of true cytotoxic anti-neoplastic potency of chemotherapeutics covalently incorporated synthetically into molecular platforms that provide properties of selective "targeted" delivery.[7,40,58,60,101-106] One of the most significant advantages of MTT vitality stain based assays and methods that apply similar reagents is that the ability to measure lethal cytotoxic anti-anti-neoplastic activity is generally considered to be superior to mearly the detection of early-stage and potentially transient cellular injury that could ultimately be reversible.

Fifth, cytotoxic anti-neoplastic potency of gemcibatine-( $\mathrm{C}_{4}$-amide $)$-[anti-HER2/neu] and gemcitabine- $\left(\mathrm{C}_{5^{-}}\right.$ methylcarbamate)-[anti-HER2/neu] immunochemotherapeutic could have been delineated in-vivo against human neoplastic xenographs in animal hosts as a model for human cancer. Effectiveness and potency of many if not most covalent immunochemotherapeutics against neoplastic cell populations (that genuinely do possess properties of selectively "targeted" chemotherapeutic delivery) is frequently higher when evaluated in-vivo compared to results acquired ex-vivo in tissue culture models utilizing the same identical cancer cell type [107-109]. Enhanced levels of covalent immunochemotherapeutic potency measured in-vivo is presumably attributable in part to induced responses by the innate immune system that includes antibody-de-pendent cell cytotoxicity (ADCC) phenomenon in concert with complementedmediated cytolysis initiated or stimulated by the formation of antigen-immunoglobulin complexes on the exterior surface membrane of "targeted" neoplastic cell types. During ADCC events immune cell types actively involved in this response release cytotoxic components that are known to additively and synergistically enhance the cytotoxic anti-neoplastic activity of conventional chemotherapeutic agents [110]. The contributions of ADCC and complement-mediated cytolysis to the in-vivo cytotoxic anti-neoplastic potency of covalent immunochemotherapeutics would be further enhanced by the additive and synergistic levels of anti-neoplastic potency produced by anti-trophic receptor monoclonal immunoglobulin when applied in dual combination with conventional chemotherapeutic agents [48,49,83,89,111-118]. Additive or synergistic interactions of this type have been detected between anti-HER2/neu when applied simultaneously in combination with cyclophosphamide [49,111], docetaxel [111], doxorubicin [49,111], etoposide [111], methotrexate [111], paclitaxel [49,111], or vinblastine [111].

Sixth, several modifications could have been made in the synthesis strategy for gemcitabine-( $\mathrm{C}_{4}$-amide)-[antiHER2/neu] and gemcitabine-( $\mathrm{C}_{5}$-methylcarbamate)-[antiHER2/neu] in order to increase the gemcitabine molar-incorporation-index. Examples in this regard include the application of gemcitabine and the covalent bond forming reagents at higher molar concentrations, implementation of smaller reaction volumes during synthesis procedures, increasing the duration of Phase I and/or Phase II synthesis schemes, and possibly altering the relative gemcitabine-to-covalent bond forming reagentto-immunoglobulin molar ratios in a manner that forces the organic chemistry reactions in a direction that increases final product yield. Unfortunately, such modifications usually also require or impose harsher reaction conditions that necessitate an acceptance for a higher risk of reduced biological activity (e.g. decreased antigen binding avidity) and substantial declines in final/total product yield $[6,108]$. Aside from overly harsh synthesis conditions, excessively high molar incorporation indexes for any chemotherapeutic agent can reduce the biological integrity of immunoglobulin fractions when the number of pharmaceutical groups introduced into the Fab' antigenbinding region becomes excessive. Such modifications can result in only modest declines in immunoreactivity (e.g. $86 \%$ for a 73:1 ratio) but disproportionately large declines in cytotoxic anti-neoplastic activity in addition to reductions in potency that can decrease to levels substantially lower than those found with non-conjugated "free" chemotherapeutic (e.g. anthracyclines) [108].

The biological integrity of the immunoglobulin component of covalent immunochemotherapeutics is critically important. It not only serves as a means of facilitating selectively "targeting" chemotherapeutic delivery, but it also initiates or induces internalization of covalent immunochemotherapeutics by mechanism of receptormediated endocytosis assuming an appropriate membrane-associated antigen has been selected as a "target" 
Cytotoxic Anti-Neoplastic Activity against Chemotherapeutic-Resistant Mammary Adenocarcinoma SKBr-3

(e.g. many carcinoma and adenocarcinoma cell types highly over-express HER2/neu and/or EGFR) [119]. Although specific data for HER2/neu and EGFR expression by mammary adenocarcinoma ( $\mathrm{SKBr}-3)$ is limited, [7] other neoplastic cell types like metastatic multiple myeloma are known to internalize and metabolize approximately $8 \times 10^{6}$ molecules of anti-CD74 monoclonal antibody per day [120]. Immunoglobulin-induced receptormediated endocytosis at membrane HER2/neu complexes can ultimately lead to increases in the intracellular concentration of selectively "targeted"/delivered chemotherapeutic that approach and exceed levels 8.5 [121] to $>100 \times$ fold greater [122] than those that can ever possibly be achieved by simple passive chemotherapeutic diffusion from out of the intravascular compartment.

The application of succinimidyl 4,4-azipentanoate in contrast to succinimidyl-4-( $N$-maleimidomethyl)-cyclohexane-1-carboxylate (SMCC), [7,80-82] N-e-maleimidocaproic acid hydrazide (EMCH), [8-10,51,52,71] or $N$-[ $p$-maleimidophenyl]-isocyanate (PMPI) [36,53-55] can facilitate greater flexibility in synthesis methods designed to increase the chemotherapeutic molar-incorporation-index during the creation of covalent immunochemotherapeutics without having to use harsher reaction conditions. The major risk of compromising the biological integrity (antigen binding avidity) of gemcitabine( $\mathrm{C}_{4}$-amide)-[anti-HER2/neu] synthesized with a UV-photoactivated gemcitabine intermediate therefore is almost entirely associated with methods devised to introduce an excessive amount of pharmaceutical (chemotherapeutic) into immunoglobulin fractions including regions of the amino acid sequence that are directly responsible for providing properties of selective "targeted" delivery (e.g. Fab antigen bindings regions of immunoglobulin or receptor binding region of ligands). Despite the general validity of the inverse relationship between chemotherapeutic molar-incorporation-index and retained biological activity (e.g. anti-HER2/neu mediated selective "targeted" delivery) and the greater potency of covalent immunochemotherapeutics with high chemotherapeutic molar incorporation indexes, it should be emphasized that mathematically the expression density for external membrane-associated "targets" appears to be one of, if not the most critically important variable that influences the cytotoxic anti-neoplastic potency of covalent immunochemotherapeutics or ligand-chemotherapeutic preparation. In this regard, it is important that external membrane-associated sites be chosen that are known to functionally undergo phenomenon analogous to receptormediated-endocytosis in order to avoid only "coating" of the external surface membrane of "targeted" cancer cell populations. Such a prerequisite is relevant assuming that the chemotherapeutic agent applied has a mechanism- of-action that is dependent upon their ability to modify the function of molecular entities within the cytosol or nucleus in order to exert a biological effect. Such a requirement would not be a prerequisite for anti-cancer agents that instead alter or disrupt the physical integrity of cancer cell membranes or the function of complexes that are an integral component of membrane structures.

\section{Acknowledgements}

The authors would like to acknowledge Mr. Tom Thompson in the Office of Agriculture Communications for assistance with the photographic illustration.

\section{REFERENCES}

[1] T. Kaneko, D. Willner, J. O. Knipe, G. R. Braslawsky, R. S. Greenfield and D. M. Vyas, "New Hydrazone Derivatives of Adriamycin and Their Immunoconjugates: A Correlation between Acid-Stability and Cytotoxicity," Bioconjugate Chemistry, Vol. 2, No. 3, 1991, pp. 133-141. doi:10.1021/bc00009a001

[2] G. Di Stefano, M. Lanza, F. Kratz, L. Merina and L. Fiume, "A Novel Method for Coupling Doxorubicin to Lactosaminated Human Albumin by an Acid Sensitive Hydrazone Bond: Synthesis, Characterization. And Preliminary Biological Properties of the Conjugate," European Journal of Pharmaceutical Sciences, Vol. 23, No. 4-6, 2004, pp. 393-397. doi:10.1016/j.ejps.2004.09.005

[3] F. Kratz, A. Warnecke, K. Scheuermann, C. Stockmar, J. Schwab, P. Lazar, P. Drückes, N. Esser, J. Drevs, D. Rognan, C. Bissantz, C. Hinderling, G. Folkers, I. Fichtner and C. Unger, "Probing the Cysteine-34 Position of Endogenous Serum Albumin with Thiol-Binding, Doxorubicin Derivatives. Improved Efficacy of an Acid-Sensitive Doxorubicin Derivative with Specific AlbuminBinding Properties Compared to That of the Parent Compound," Journal of Medicinal Chemistry, Vol. 45, No. 25, 2002, pp. 5523-5533. doi:10.1021/jm020276c

[4] C. Unger, B. Häring, M. Medinger, J. Drevs, S. Steinbild, F. Kratz and K. Mross, "Phase I and Pharmacokinetic Study of the (6-Maleimidocaproyl) Hydrazone Derivative of Doxorubicin," Clinical Cancer Research, Vol. 13, No. 16,2007, pp. 4858-4866. doi:10.1158/1078-0432.CCR-06-2776

[5] C. Mazuel, J. Grove, G. Gerin and K. P. Keenan, "HPLCMS/MS Determination of a Peptide Conjugate Prodrug of Doxorubicin, and Its Active Metabolites, Leucine-Doxorubicin and Doxorubicin, in Dog and Rat Plasma," Journal of Pharmaceutical and Biomedical Analysis, Vol. 33, No. 5, 2003, pp. 1093-1102. doi:10.1016/S0731-7085(03)00434-5

[6] R. S. Greenfield, T. Kaneko, A. Daues, M. A. Edson, K. A. Fitzgerald, L. J. Olech, J. A. Grattan, G. L. Spitalny and G. R. Braslawsky, "Evaluation In-Vitro of Adriamycin Immunoconjugates Synthesized Using an Acid-Sensitive Hydrazone Linker," Cancer Research, Vol. 50, No. 20, 1990, pp. 6600-6607. 
[7] C. P. Coyne, M. Ross, J. Bailey and T. Jones, "Dual Potency Anti-HER2/neu and Anti-EGFR AnthracyclineImmunoconjugates in Chemotherapeutic-Resistant Mammary Carcinoma Combined with Cyclosporin-A and Verapamil P-Glycoprotein Inhibition," Journal of Drug Targeting, Vol. 17, No. 6, 2009, pp. 474-489. doi:10.1080/10611860903012802

[8] A. Lau, G. Berube, C. H. J. Ford and M. Gallant, "Novel Doxorubicin-Monoclonal Anti-Carcinoembryonic Antigen Antibody Immunoconjugate Activity In-Vivo" Bioorganic and Medicinal Chemistry, Vol. 3, No. 10, 1995, pp. 1305-1312. doi:10.1016/0968-0896(95)00126-2

[9] M. Kruger, U. Beyer, P. Schumacher, C. Unger, H. Zahn and F. Kratz, "Synthesis and Stability of Four Maleimide Derivatives of the Anti-Cancer Drug Doxorubicin for the Preparation of Chemoimmunoconjugates," Chemical \& Pharmaceutical Bulletin, Vol. 45, No. 2, 1997 pp. 399401. doi:10.1248/cpb.45.399

[10] D. Y. Furgeson, M. R. Dreher and A. Chilkoti, "Structural Optimization of a 'Smart' Doxorubicin-Polypeptide Conjugate for Thermally Targeted Delivery to Solid Tumors," Journal of Controlled Release, Vol 110, No. 2, 2006, pp. 362-369. doi:10.1016/j.jconrel.2005.10.006

[11] J. F. Liang and V. C. Yang, "Synthesis of Doxorubicin-Peptide Conjugate with Multidrug Resistant Tumor Cell Killing Activity," Bioorganic and Medicinal Chemistry Letters, Vol. 15, No. 22, 2005, pp. 5071-5075. doi:10.1016/j.bmcl.2005.07.087

[12] M. Sirova, J. Strohalm, V. Subr, D. Plocova, P. Rossmann, T. Mrkvan, K. Ulbrich and B. Rihova, "Treatment with HPMA Copolymer-Based Doxorubicin Conjugate Containing Human Immunoglobulin Induces Long-Lasting Systemic Anti-Tumor Immunity in Mice," Cancer Immunol Immunother, Vol. 56, No. 1, 2007, pp. 35-47. doi:10.1007/s00262-006-0168-0

[13] B. K. Wong, D. Defeo-Jones, R. E. Jones, V. M. Garsky, D. M. Feng, A. Oliff, M. Chiba, J. D. Ellis and J. H. Lin, "PSA-Specific and Non-PSA-Specific Conversion of a PSA-Targeted Peptide Conjugate of Doxorubicin to Its Active Metabolite," Drug Metabolism and Disposition, Vol. 29, No. 3, 2001, pp. 313-318.

[14] G. L. Bidwell, A. N. Davis, I. Fokt, W. Priebe and D. Raucher, "A Thermally Targeted Elastin-Like Polypeptide-Doxorubicin Conjugate Overcomes Drug Resistance," Investigational New Drugs, Vol. 25, No. 4, 2007, pp. 313-326. doi:10.1007/s10637-007-9053-8

[15] K. A. Ajaj, R. Graeser, I. Fichtner and F. Kratz, "In-Vitro and In-Vivo Study of an Albumin-Binding Prodrug of Doxorubicin That Is Cleaved by Cathepsin B," Cancer Chemotherapy and Pharmacology, Vol. 64, No. 2, 2009, pp. 413-418. doi:10.1007/s00280-009-0942-8

[16] C. Ryppa, H. Mann-Steinberg, I. Fichtner, H. Weber, R. Satchi-Fainaro, M. L. Biniossek and F. Kratz, "In-Vitro and In-Vivo Evaluation of Doxorubicin Conjugates with the Divalent Peptide E-[c(RGDfK)2] That Targets Integrin aVb3," Bioconjugate Chemistry, Vol. 19, No. 7, 2008 pp. 1414-1422. doi:10.1021/bc800117r

[17] Y. F. Huang, D. Shangguan, H. Liu, J. A. Phillips, X.
Zhang, Y. Chen and W. Tan, "Molecular Assembly of an Aptamer-Drug Conjugate for Targeted Drug Delivery to Tumor Cells," A European Journal of Chemical Biology, Vol. 10, No. 5, 2009, pp. 862-868. doi:10.1002/cbic. 200800805

[18] Y. Ren, D. Wei and X. Zhan, "Inhibition of P-Glycoprotein and Increasing of Drug-Sensitivity of a Human Carcinoma Cell Line (KB-A-1) by an Anti-Sense Oligodeoxynucleotide-Doxorubicin Conjugate In-Vitro," Biotechnology and Applied Biochemistry, Vol. 41, No. 2, 2005, pp. 137-143. doi:10.1042/BA20040058

[19] Y. Ren, X. Zhan, D. Wei and J. Liu, "In-Vitro Reversal MDR of Human Carcinoma Cell Line by an Antisense Oligodeoxynucleotide-Doxorubicin Conjugate," Biomedicine \& Pharmacotherapy, Vol. 58. No. 9, 2004, pp. 520526.

[20] L. Kovar, T. Etrych, M. Kabesova, V. Subr, D. Vetvicka, O. Hovorka, J. Strohalm, J. Sklenar, P. Chytil, K. Ulbrich and B. Rihova, "Doxorubicin Attached to HPMA Copolymer via Amide Bond Modifies the Glycosylation Pattern of EL4 Cells," Tumor Biology, Vol. 31. No. 4, 2010, pp 233-242. doi:10.1007/s13277-010-0019-7

[21] T. Lammers, V. Subr, K. Ulbrich, P. Peschke, P. E. Huber, W. E. Hennink and G. Storm, "Simultaneous Delivery of Doxorubicin and Gemcitabine to Tumors in Vivo Using Prototypic Polymeric Drug Carriers," Biomaterials, Vol. 30, No. 20, 2009, pp. 3466-3475. doi:10.1016/j.biomaterials.2009.02.040

[22] H. Krakovicova, T. Ethch and K. Ulbrich, "HPMA-Based Polymerconjugates with Drug Combinations," European Journal of Pharmacology, Vol. 37. No. 3-4, 2009, pp. 4050-4412.

[23] N. Cao and S. S. Feng, "Doxorubicin Conjugated to D-Alpha-Tocopheryl Polyethylene Glycol 1000 Succinate (TPGS): Conjugation Chemistry, Characterization, InVitro and In-Vivo Evaluation," Biomaterials, Vol. 29, No. 28, 2008, pp. 3856-3865.

doi:10.1016/j.biomaterials.2008.05.016

[24] P. C. Rodrigues, U. Beyer, P. Schumacher, T. Roth, H. H. Fiebig, C. Unger, L. Messori, P. Orioli, D. H. Paper, R. Mülhaupt and F. Kratz, "Acid-Sensitive Polyethylene Glycol Conjugates of Doxorubicin: Preparation, In-Vitro Efficacy and Intracellular Distribution," Bioorganic \& Medicinal Chemistry, Vol. 7, No. 11, 1999, pp. $2517-$ 2524. doi:10.1016/S0968-0896(99)00209-6

[25] F. Kratz, "Albumin as a Drug Carrier: Design of Prodrugs, Drug Conjugates and Nanoparticles," Journal of Controlled Release, Vol. 132, No. 3, 2008, pp. 171-183. doi:10.1016/j.jconrel.2008.05.010

[26] K. Inoh, H. Muramatsu, S. Torii, S. Ikematsu, M. Oda, H. Kumai, S. Sakuma, T. Inui, T. Kimura and T. Muramatsu, "Doxorubicin-Conjugated Anti-Midkine Monoclonal Antibody as a Potential Anti-Tumor Drug," Japanese Journal of Clinical Oncology, Vol. 36, No. 4, 2006, pp. 207-211. doi:10.1093/ijco/hyl004

[27] G. L. Griffiths, M. J. Mattes, R. Stein, S. V. Govindan, I. D. Horak, H. J. Hansen and D. M. Goldenberg, "Cure of SCID Mice Bearing Human B-Lymphoma Xenografts by 
an Anti-CD74 Antibody-Anthracycline Drug Conjugate," Clinical Cancer Research, Vol. 9, No. 17, 2003, pp. 6567-6571.

[28] P. Sapra, R. Stein, J. Pickett, Z. Qu, S. Govindan, V, T. M. Cardillo, H. J. Hanson, I. D. Horak, G. L. Griffiths and D. M. Goldenberg, "Anti-CD74 Antibody-Doxorubicin Conjugate, IMMU-110, in a Human Multiple Myeloma Xenograph and in Monkeys," Clinical Cancer Research, Vol. 11 , No. 14, 2005, pp. 5257-5264. doi:10.1158/1078-0432.CCR-05-0204

[29] H. M. Yang and R. A. Reisfeld, "Doxorubicin Conjugated with Monoclonal Antibody Directed to a Human Melanoma-Associated Proteoglycan Suppresses Growth of Established Tumor Xenografts in Nude Mice," Proceedings of the National Academy of Sciences, Vol. 85, 1988, pp. 1189-1193. doi:10.1073/pnas.85.4.1189

[30] P. A. Trail, D. Willner, S. J. Lasch, A. J. Henderson, S. Hofstead, A. M. Casazza, R. A. Firestone, I. Hellstrom and K. E. Hellstrom, "Cure of Xenografted Human Carcinomas by BR96-Doxorubicin Immunoconjugates," Science, Vol. 261, No. 5118, 1993, pp. 212-215. doi:10.1126/science. 8327892

[31] E. Diener, U. Diner, A. Sinha, S. Xie and R. Vergidis, "Specific Immunosuppression by Immunotoxins Containing Daunomycin," Science, Vol. 231, No. 4734, 1986, pp. 148-150. doi:10.1126/science. 3484557

[32] R. O. Dillman, D. E. Johnson, J. Ogden and D. Beidler, "Significance of Antigen, Drug, and Tumor Cell Targets in the Preclinical Evaluation of Doxorubicin, Daunorubicin, Methotrexate, and Mitomycin-C Monoclonal Antibody Immunoconjugates," Molecular Biotherapy, Vol. 1, 5, 1989, pp. 250-255.

[33] M. Page, D. Thibeault, C. Noel and L. Dumas, "Coupling a Preactivated Daunorubicin Derivative to Antibody. A New Approach," Anticancer Research, Vol. 10, No. 2A, 1990, pp. 353-357.

[34] J. Reményi, B. Balázs, S. Tóth, A. Falus, G. Tóth and F. Hudecz, "Isomer-Dependent Daunomycin Release and in Vitro Antitumour Effect of Cis-Aconityl-Daunomycin," Biochemical and Biophysical Research Communications, Vol. 303, No. 2, 2003, pp. 556-561. doi:10.1016/S0006-291X(03)00394-2

[35] J. R. Ogden, K. Leung, S. A. Kunda, M. W. Telander, B. P. Avner, S. K. Liao, G. B. Thurman and R. K. Oldham, "Immunoconjugates of Doxorubicin and Murine Antihuman Breast Carcinoma Monoclonal Antibodies Prepared Via an N-Hydroxysuccinimide Active Ester Intermediate of Cis-Aconityl-Doxorubicin: Preparation and in Vitro Cytotoxicity," Molecular Biotherapy, Vol 1, No. 3, 1989, pp. $170-174$.

[36] C. P. Coyne, T. Jones and T. Pharr, "Synthesis of a Covalent Gemcitabine-(Carbamate)-[Anti-HER2/neu] immunochemotherapeutic and Cytotoxic Anti-Neoplastic Activity against Chemotherapeutic-Resistant SKBr-3 Mammary Carcinoma," Bioorganic and Medicinal Chemistry, Vol. 19, No. 1, 2011, pp. 67-76. doi:10.1016/j.bmc.2010.11.046

[37] A. I. Shamseddine, M. J. Khalifeh, F. H. Mourad, A. A.
Chehal, A. Al-Kutoubi, J. Abbas, M. Z. Habbal, L. A Malaeb and A. B. Bikhazi, "Comparative Pharmacokinetics and Metabolic Pathway of Gemcitabine during Intravenous and Intra-Arterial Delivery in Unresectable Pancreatic Cancer Patients," Clinical Pharmacokinetics, Vol. 44, No. 9, 2005, pp. 957-967. doi:10.2165/00003088-200544090-00005

[38] E. Giovannetti, A. C. Laan, E. Vasile, C. Tibaldi, S. Nannizzi, S Ricciardi, A. Falcone, R. Danesi and G. J. Peters, "Correlation between Cytidine Deaminase Genotype and Gemcitabine Deamination in Blood Samples," Nucleosides Nucleotides Nucleic Acids, Vol. 27, No. 6, 2008, pp. 720-725. doi:10.1080/15257770802145447

[39] J. A. Gilbert, O. E. Salavaggione, Y. Ji, L. L. Pelleymounter, B. W. Eckloff, E. D. Wieben, M. M. Ames and R. M. Weinshilboum, "Gemcitabine Pharmacogenomics: Cytidine Deaminase and Deoxycytidylate Deaminase Gene Resequencing and Functional Genomics," Clinical Cancer Research, Vol. 12, No. 6, 2006, pp. 1794-1803. doi:10.1158/1078-0432.CCR-05-1969

[40] R. L. Alexander, B. T. Greene, S. Torti and V. G. L. Kucera, "A Novel Phospholipid Gemcitabine Conjugate Is Able to Bypass Three Drug-Resistance Mechanisms," Cancer Chemotherapy and Pharmacology, Vol. 56, No. 1, 2005, pp. 15-21. doi:10.1007/s00280-004-0949-0

[41] R. J. Pietras, M. D. Pegram, R. S. Finn, D. A. Maneval and D. J. Slamon, "Remission of Human Breast Cancer Xenografts on therapy with Humanized Monoclonal Antibody to HER-2 Receptor and DNA-Reactive Drugs," Oncogene, Vol. 17, No. 17, 1998, pp. 2235-2249. doi:10.1038/sj.onc.1202132

[42] R. Marches and J. W. Uhr, "Enhan Cement of the p27Kip1-Mediated Antiproliferative Effect of Trastuzumab (Herceptin) on HER2-Overexpressing Tumor Cells," International Journal of Cancer, Vol. 112, No. 3, 2004, pp. 492-501. doi:10.1002/ijc. 20378

[43] M. Sliwkowski, X, J. A. Lofgren, G. D. Lewis, T. E. Hotaling, B. M. Fendly and J. A. Fox, "Nonclinical Studies Addressing the Mechanism of Action of Trastuzumab (Herceptin)," Seminars in Oncology, Vol. 26, Suppl. 12 1999, pp. 60-70.

[44] N. U. Lin, L. A. Carey, M. C. Liu, J. Younger, S. E. Come, M. Ewend, G. Harris, E. Bullitt, A. D. Van den Abbeele, J. W. Henson, X. Li, R. Gelman, H. J. Burstein, E. Kasparian, D. G. Kirsch, A. Crawford, F. Hochberg and E. P. Winer, "Phase II Trial of Lapatinib for Brain Metastases in Patients with Human Epidermal Growth Factor Receptor 2-Positive Breast Cancer," Journal of Clinical Oncology, Vol. 26, No. 12, 2008, pp. 1993-1999. doi:10.1200/JCO.2007.12.3588

[45] M. A. Cobleigh, C. L. Vogel, D. Tripathy, N. J. Robert, S. Scholl, L. Fehrenbacher, J. Wolter, V. Paton, S. Shak, G. Lieberman and D. J. Slamon, "Multinational Study of the Efficacy and Safety of Humanized Anti-HER2 Monoclonal Antibody in Women Who Have HER2-Overexpressing Metastatic Breast Cancer That Has Progressed after Chemotherapy for Metastatic Disease," Journal of Clinical Oncology, Vol. 17, No. 9, 1999, pp. 2639-2648.

[46] C. L. Vogel, M. A. Cobleigh, D. Tripathy, J. C. Gutheil, 
L. N. Harris, L. Fehrenbacher, D. J. Slamon, M. Murphy, W. F. Novotny, M. Burchmore, S. Shak, S. J. Stewart and M. Press, "Efficacy and Safety of Trastuzumab as a Single Agent in First-Line Treatment of HER2-Overexpressing Metastatic Breast Cancer," Journal of Clinical Oncology, Vol. 20, No. 3, 2002, pp. 719-726. doi:10.1200/JCO.20.3.719

[47] G. D. Lewis Phillips, G. Li, D. L. Dugger, L. M. Crocker, K. L. Parsons, E. Mai, W. A. Blättler, J. M. Lambert, R. Chari, V, R. J. Lutz, W. L. Wong, F. S. Jacobson, H. Koeppen, R. H. Schwall, S. R. Kenkare-Mitra, S. D. Spencer and M. X. Sliwkowski, "Targeting HER2-Positive Breast Cancer with Trastuzumab-DM1, an AntibodyCytotoxic Drug Conjugate," Cancer Research, Vol. 68, No. 22, 2008, pp. 9280-9290. doi:10.1158/0008-5472.CAN-08-1776

[48] J. A. García-Sáenz, M. Martín, A. Calles, C. Bueno, L. Rodríguez, J. Bobokova, A. Custodio, A. Casado and E. Díaz-Rubio, "Bevacizumab in Combination with Metronomic Chemotherapy in Patients with Anthracycline- and Taxane-Refractory Breast Cancer," Journal of Chemotherapy, Vol. 20, No. 5, 2008, pp. 632-639.

[49] D. J. Slamon, B. Leyland-Jone, S. Shak, H. Fuchs, V. Paton, A. Bajamonde, T. Fleming, W. Eiermann, J. Wolter and M. Pegram, "Use of Chemotherapy plus Monoclonal Antibody against HER2 for Metastatic Breast Cancer That Overexpress HER2," The New England Journal of Medicine, Vol. 344, No. 11, 2001, pp. 786-792. doi:10.1056/NEJM200103153441101

[50] U. Beyer, T. Roth, P. Schumacher, G. Maier, A. Unold, A. W. Frahm, H. H. Fiebig, C. Unger and F. Kratz, "Synthesis and In-Vitro Efficacy of Transferring Conjugates of the Anticancer Drug Chlorambucil," Journal of Medicinal Chemistry, Vol. 41, No. 15, 1998, pp. 2701-2708. doi:10.1021/jm9704661

[51] L. C. Xu, M. Nakayama, K. Harada, A. Kuniyasu, H. Nakayama, S. Tomiguchi, A. Kojima, M. Takahashi, M. Ono, Y. Arano, H. Saji, Z. Yao, H. Sakahara, J. Konishi and Y. Imagawa, "Bis(Hydroxamamide)-Based Bifunctional Chelating Agent for 99mTc Labeling of Polypeptides," Bioconjugate Chemistry, Vol. 10, No. 1, 1999, pp. 9-17. doi:10.1021/bc980024j

[52] Y. Arano, T. Uezono, H. Akizawa, M. Ono, K. Wakisaka, M. Nakayama, H. Sakahara, J. Konishi and A. Yokoyama, "Reassessment of Diethylenetriaminepentaacetic Acid (DTPA) as a Chelating Agent for Indium-111 Labeling of Polypeptides Using a Newly Synthesized Monoreactive DTPA Derivative," Journal of Medicinal Chemistry, Vol. 39 No. 18, 1996, pp. 3451-3460. doi:10.1021/jm950949+

[53] M. E. Annunziato, U. S. Patel, M. Ranade and P. S. Palumbo, "p-Maleimidophenyl Isocyanate: A Novel Heterbifunctional Linker for Hydroxyl to Thiol Coupling," Bioconjugate Chemistry, Vol. 4, No. 3, 1993, pp. 212-218. doi: $10.1021 / \mathrm{bc} 00021 \mathrm{a} 005$

[54] Q. Liu, J. R. de Wijn and C. A. van Blitterswijk, "Covalent Bonding of PMMA, PBMA, and Poly(HEMA) to Hydroxyapatite Particles," Journal of Biomedical Materials Research, Vol. 40, No. 2, 1998, pp. 257-263. doi:10.1002/(SICI)1097-4636(199805)40:2<257::AID-JB

\section{$\underline{\mathrm{M} 10>3.0 . \mathrm{CO} ; 2-\mathrm{J}}$}

[55] X. L. Wang, Y. Huang, J. Zhu, Y. B. Pan, R. He and Y. Z. Wang, "Chitosan-Graft Poly(p-Dioxanone) Copolymers: Preparation, Characterization, and Properties," Carbohydrate Research, Vol. 344, No. 6, 2009, pp. 801-807.

[56] S. M. Ali, A. R. Khan, M. U. Ahmad, P. Chen, S. Sheikh and I. Ahmad, "Synthesis and Biological Evaluation of Gemcitabine-Lipid Conjugate (NEO6002)," Bioorganic $\&$ Medicinal Chemistry Letters, Vol. 15, No. 10, 2005, pp. 2571-2574. doi:10.1016/j.bmcl.2005.03.046

[57] P. Chen, P. Y. Chien, A. R. Khan, S. Sheikh, S. M. Ali, M. U. Ahmad and I. Ahmad, "In-Vitro and in-Vivo Anti-Cancer Activity of a Novel Gemcitabine-Cardiolipin Conjugate," Anticancer Drugs, Vol. 17, No. 1, 2006, pp. 53-61. doi:10.1097/01.cad.0000185182.80227.48

[58] L. V. Kiew, S. K. Cheong, K. Sidik and L. Y. Chung, "Improved Plasma Stability and Sustained Release Profile of Gemcitabine via Polypeptide Conjugation," International Journal of Pharmaceutics, Vol. 391, No. 1-2, 2010, pp. 212-220. doi:10.1016/i.ijpharm.2010.03.010

[59] P. Guo, J. Ma, S. Li, Z. Guo, A. L. Adams and J. M. Gallo, "Targeted Delivery of a Peripheral Benzodiazepine Receptor Ligand-Gemcitabine Conjugate to Brain Tumors in a Xenograft Model," Cancer Chemotherapy and Pharmacology, Vol. 48, No. 2 , 2001, pp. 169-176. doi:10.1007/s002800100284

[60] R. L. Alexander, S. L. Morris-Natschke, K. S. Ishaq, R. A. Fleming and G. L. Kucera, "Synthesis of Cytotoxic Activity of Two Novel 1-Dodecylthio-2-Decyloxypropyl3-Phophatidic Acid Conjugates with Gemcitabine and Cytosine Arabinoside," Journal of Medicinal Chemistry, Vol. 46, 19, 2003, pp. 4205-4208. doi:10.1021/jm020571x

[61] Z. Guo and J. M. Gallo, "Selective Protection of 2',2'Difluorodexoycytidine (Gemcitabine)," The Journal of Organic Chemistry, Vol. 64, No. 22, 1999, pp. 8319-8322. doi:10.1021/j09911140

[62] P. A. Trail, D. Willner, J. Knipe, A. J. Henderson, S. J. Lasch, M. E. Zoeckler, M. D. TrailSmith, T. W. Doyle, H. D. King, A. M. Casazza, J. Brown, S. J. Hofstead, R. S. Greenfield, R. A. Firestone, K. Mosure, K. F. Kadow, M. B. Yang, K. E. Hellstrom and I. Hellstrom, "Effect of linker Variation on the Stability, Potency and Efficacy of Carcinoma-Reactive BR64-Doxorubicin Immunoconjugates," Cancer Research, Vol. 57, No. 1, 1997, pp. 100 105.

[63] T. Etrych, T. Mrkvan, B. Ríhová and K. Ulbrich, "StarShaped Immunoglobulin-Containing HPMA-Based Conjugates with Doxorubicin for Cancer Therapy," Journal of Controlled Release, Vol. 122, No. 1, 2007, pp. 31-38. doi:10.1016/j.jconrel.2007.06.007

[64] J. Liu, H. Zhao, K. J. Volk, S. E. Klohr, E. H. Kerns and M. S. Lee, "Analysis of Monoclonal Antibody and Immunoconjugate Digests by Capillary Electrophoresis and Capillary Liquid Chromatography," Journal of Chromatography A, Vol. 735, No. 1-2, 1996, pp. 357-366. doi:10.1016/0021-9673(95)01054-8

[65] F. Kratz, G. Ehling, H.-M. Kauffmann and C. Unger, 
"Acute and Repeat-Dose Toxicity Studies of the (6Maleimidocaproyl)Hydrazone Derivative of Doxorubicin (DOXO-EMCH), an Albumin-Binding Prodrug of the Anticancer Agent Doxorubicin," Human \& Experimental Toxicology, No. 26, No. 1, 2007, pp. 19-35. doi:10.1177/0960327107073825

[66] D. Lebrecht, A. Geist, U. P. Ketelsen, J. Haberstroh, B. Setzer, F. Kratz and U. Walker, "The 6-Maleimidocaproyl Hydrazone Derivative of Doxorubicin (Doxoemch) Is Superior to Free Doxorubicin with Respect to Cardiotoxicity and Mitochondrial Damage," International Journal of Cancer, Vol. 120, No. 4, 2006, pp. 927-934.

[67] F. Kratz, U. Beyer, P. Collery, F. Lechenault, A. Cazabat, P. Schumacher, U. Falken and C. Unger, "Preparation, Characterization and In-Vitro Efficacy of Albumin Conjugates of Doxorubicin," Biological and Pharmaceutical Bulletin, Vol. 21, No. 1, 1998, pp. 56-61. doi:10.1248/bpb.21.56

[68] F. Kratz, U. Beyer, T. Roth, N. Tarasova, P. Collery, F. Lechenault, A. Cazabat, P. Schumacher, C. Unger and U. Falken, "Transferrrin Conjugates of Doxorubicin: Synthesis, Characterization, Cellular Uptake, and In-Vitro Efficacy," Journal of Pharmaceutical Sciences, Vol. 87, No. 3, 1998, pp. 338-346. doi:10.1021/js970246a

[69] M. N. Kirstein, I. Hassan, D. E. Guire, D. R. Weller, J. W. Dagit, J. E. Fisher and R. P. Remmel, "High-Performance Liquid Chromatographic Method for the Determination of Gemcitabine and 2',2'-Difluorodeoxyuridine in Plasma and Tissue Culture Media," Journal of Chromatography B: Biomedical Sciences and Applications, Vol. 835, No. 1-2, 2006, pp. 136-142. doi:10.1016/j.jchromb.2006.03.023

[70] V. Reichelova, F. Albertioni and J. Liliemark, "Determination of 2-Chloro-2'-Deoxyadenosine Nucleotides in Leukemic Cells by Ion-Pair High-Performance Liquid Chromatography," Journal of Chromatography B: Biomedical Sciences and Applications, Vol. 682, No. 1, 1996, pp. 115-123. doi:10.1016/0378-4347(96)00048-5

[71] C. P. Coyne, T. Jones, A. Sygula, J. Bailey and L. Pinchuk, "Epirubicin-[Anti-HER2/neu] Synthesized with an Epirubicin-( $\mathrm{C}_{13}$-Imino)-EMCS Analog: Anti-Neo-plastic Activity against Chemotherapeutic-Resistant SKBr-3 Mammary Carcinoma in Combination with Organic Selenium," Journal of Cancer Therapy, Vol. 2, No. 1, 2011, pp. 22-39. doi:10.4236/jct.2011.21004

[72] C. P. Coyne, T. Jones and R. Bear, "Synthesis of Epirubicin-( $\mathrm{C}_{3}$-amide)-[Anti-HER2/neu] utilizing a UV-Photoactivated Epirubicin Intermediate," Cancer Biotherapy and Radiopharmaceuticals, Vol. 27, No. 1, 2012, pp. 4155. doi:10.1089/cbr.2011.1097

[73] U. Beyer, B. Rothen-Rutishauser, C. Unger, H. WunderliAllenspach and F. Kratz, "Difference in the Intracellular Distribution of Acid-Sensitive Doxorubicin-Protein Conjugates in Comparison to Free and Liposomal-Formulated Doxorubicin as Shown by Confocal Microscopy," Pharmaceutical Research, Vol. 18, No. 1, 2001, pp. 29-38. doi:10.1023/A:1011018525121

[74] J. A. Sinkule, S. T. Rosen and J. A. Radosevich, "Mono- clonal Antibody 44-3A6 Doxorubicin Immunoconjugates: Comparative in Vitro Anti-Tumor Efficacy of Different Conjugation Methods," Tumour Biology, Vol. 12, No. 4, 1991, pp 198-206. doi:10.1159/000217705

[75] G. P. Sivam, P. J. Martin, R. A. Reisfeld and B. M. Mueller, "Therapeutic Efficacy of a Doxorubicin Immunoconjugate in a Preclinical Model of Spontaneous Metastatic Human Melanoma," Cancer Research, Vol. 55, No. 11, 1995, pp. 2352-2356.

[76] R. L. Alexander and G. L. Kucera, "Lipid nucleoside Conjugates for the Treatment of Cancer," Current Pharmaceutical Design, Vol. 11, No. 9, 2005, pp. 1079-1089. doi:10.2174/1381612053507602

[77] P. Lagisetty, P. Vilekar and V. Awasthi, "Synthesis of Radiolabeled Cytarabine Conjugates," Bioorganic \& Medicinal Chemistry Letters, Vol. 19, No. 16, 2009, pp. 4764 4767. doi:10.1016/j.bmcl.2009.06.056

[78] F. Castelli, M. G. Sarpietro, M. Ceruti, F. Rocco and L. Cattel, "Characterization of Lipophilic Gemcitabine Prodrug-Liposomal Membrane Interaction by Differential Scanning Calorimetry," Molecular Pharmaceutics, Vol. 3, No. 6, 2006, pp. 737-744. doi:10.1021/mp060059y

[79] L. H. Reddy, C. Dubernet, S. L. Mouelhi, P. E. Marque, D. Desmaele and P. Couvreur, "A New Nanomedicine of Gemcitabine Displays Enhanced Anticancer Activity in Sensitive and Resistant Leukemia Types," Journal of Controlled Release, Vol. 124, No. 1-2, 2007, pp. 20-27. doi:10.1016/i.jconrel.2007.08.018

[80] S. H. Frost, H. Jensen and S. Lindegren, "In-Vitro Evaluation of Avidin Antibody Pretargeting Using [211At]-Labeled and Biotinylated Poly-L-Lysine as Effector Molecule," Cancer, Vol. 116, No. S4, 2010, pp. 1101-1110. doi:10.1002/cncr.24798

[81] H. Karacay, R. M. Sharkey, S. Govindan, V, W. J. McBride, D. M. Goldenberg, H. J. Hansen and G. L. Griffiths, "Development of a Streptavidin-Anti-Carcinoembryonic Antigen Antibody, Radiolabeled Biotin Pretargeting Method for Radioimmunotherapy of Colorectal Cancer. Reagent Development," Bioconjugate Chemistry, Vol. 8, No. 4, 1997, pp. 585-594. doi: $10.1021 /$ bc970102n

[82] A. Lau, G. Bérubéand and C. H. ford, "Conjugation of Doxorubicin to Monoclonal Anti- Carcinoembryonic Antigen Antibody via Novel Thiol-Directed Cross-Linking Reagents," Bioorganic \& Medicinal Chemistry, Vol. 3, No. 10, 1995, pp. 1299-1304. doi:10.1016/0968-0896(95)00125-Z

[83] A. K. Fry, K. F. Schilke, J. McGuire and K. E. Bird, "Synthesis and Anticoagulant Activity of Heparin Immobilized 'End-On' to Polystyrene Microspheres Coated with End-Group Activated Polyethylene Oxide," Journal of Biomedical Materials Research Part B: Applied Biomaterials, Vol. 94, No. 1, 2010, pp. 187-195.

[84] M. H. Vingerhoeds, H. J. Haisma, S. O. Belliot, R. H. Smit, D. J. Crommelin and G. Storm, "Immunoliposomes as Enzyme-Carriers (Immuno-Enzymosomes) for Antibody-Directed Enzyme Prodrug Therapy (ADEPT): Optimization of Prodrug Activating Capacity," Pharmaceu- 
tical Research, Vol. 13, No. 4, 1996, pp. 604-610. doi:10.1023/A:1016010524510

[85] K. Fujiwara, M. Yasuno and T. Kitagawa, "Novel Preparation Method of Immunogen for Hydrophobic Hapten, Enzyme Immunoassay for Daunomycin and Adriamycin," Journal of Immunological Methods, Vol. 45, No. 2, 1981, pp. 195-203. doi:10.1016/0022-1759(81)90213-1

[86] Y. Wang, X. Liu and D. J. Hnatowich, "An improved Synthesis of NHS-MAG3 for Conjugation and Radiolabeling of Biomolecules with [99mTc] at Room Temperature," Nature Protocols, Vol. 2, No. 4, 2007, pp. 972-978. doi:10.1038/nprot.2007.144

[87] P. Joshi, K. F. Schilke, A. Fry, J. McGuire and K. Bird, "Synthesis and Evaluation of Heparin Immobilized 'SideOn' to Polystyrene Microspheres Coated with End-Group Activated Polyethylene Oxide," International Journal of Biological Macromolecules, Vol. 47, No. 2 , 2010, pp. 98103. doi:10.1016/j.ijbiomac.2010.05.015

[88] J. Vega, S. Ke, Z. Fan, S. Wallace, C. Charsangavej and C. Li, "Targeting Doxorubicin to Epidermal Growth Factor Receptors by Site-Specific Conjugation of C225 to Poly(L-Glutamic Acid) through a Polyethylene Glycol Spacer," Pharmaceutical Research, Vol. 20, No. 5, 2003 pp. 826-832. doi:10.1023/A:1023454107190

[89] R. Jin, L. S. Moreira Teixeira, A. Krouwels, P. J. Dijkstra, C. A. van Blitterswijk, M. Karperien and J. Feijen, "Synthesis and Characterization of Hyaluronic Acid-Poly (Ethylene Glycol) Hydrogels via Michael addition: An Injectable Biomaterial for Cartilage Repair," Acta Biomater, Vol. 6, No. 6, 2010, pp. 1968-1977. doi:10.1016/j.actbio.2009.12.024

[90] J. Morales-Sanfrutos, A. Megia-Fernandez, F. Hernandez-Mateo, M. D. Giron-Gonzalez, M. D. R. S. Gonzalez and F. Santoyo-Gonzalez, "Alkyl Sulfonyl Derivatized PAMAM-G2 Dendrimers as Nonviral Gene Delivery Vectors with Improved Transfection Efficiencies," Organic \& Biomolecular Chemistry, Vol. 9, No. 3, 2011, pp. 851-864. doi:10.1039/c0ob00355g

[91] M. Haas, F. Moolenaar, A. Elsinga, E. A. Van der Wouden, P. E. De Jong, D. K. F. Meijer and D. D. Zeeuw, "Targeting of Doxorubicin to the Urinary Bladder of the Rat Shows Increased Cytotoxicity in the Bladder Urine Combined with an Absence of Renal Toxicity," Journal of Drug Targeting, Vol. 10, No. 1, 2002, pp. 81-89. doi:10.1080/10611860290007568

[92] American Cancer Society, "Cancer Facts and Figures 2004," American Cancer Society, 2004, pp. 1-60.

[93] H. M. Yang and R. A. Reisfeld, "Pharmacokinetics and Mechanism of Action of a Doxorubicin-Monoclonal Antibody 9. 2. 27 Conjugate Directed to a Human Melanoma Proteoglycan," Journal of the National Cancer Institute, Vol. 80, No. 14, 1988, pp. 1154-1159. doi:10.1093/jnci/80.14.1154

[94] S. Lutsenko, V, N. B. Feldman and S. E. Severin, "Cytotoxic and Antitumor Activities of Doxorubicin Conjugates with the Epidermal Growth Factor and Its Receptor-Binding Fragment," Journal of Drug Targeting, Vol. 10, No. 7, 2002, pp. 567-571.

\section{doi:10.1080/1061186021000038058}

[95] C. W. Michalski, M. Erkan, D. Sauliunaite, T. Giese, R. Stratmann, C. Sartori, N. A. Giese, H. Friess and J. Kleeff, "Ex-Vivo Chemosensitivity Testing and Gene Expression Profiling Predict Response Towards Adjuvant Gemcitabine Treatment in Pancreatic Cancer," British Journal of Cancer, Vol. 99, No. 5, 2008, pp. 760-767. doi:10.1038/sj.bjc. 6604528

[96] T. Hoang, K. Kim, A. Jaslowski, P. Koch, P. Beatty, J. McGovern, M. Quisumbing, G. Shapiro, R. Witte and J. H. Schiller, "Phase II Study of Second-Line Gemcitabine in Sensitive or Refractory Small Cell Lung Cancer," Lung Cancer, Vol. 42, No. 1, 2003, pp. 97-102. doi:10.1016/S0169-5002(03)00273-3

[97] J. Bierau, A. H. van Gennip, R. Leen, R. Meinsma, H. N. Caron and A. B. van Kuilenburg, "Cyclopentenyl Cytosine-Induced Activation of Deoxycytidine Kinase Increases Gemcitabine Anabolism and Cytotoxicity in Neuroblastoma," Cancer Chemotherapy and Pharmacology, Vol. 57, No. 1, 2006, pp. 105-113. doi:10.1007/s00280-005-0005-8

[98] V. Santini, G. D’Ippolito, P. A. Bernabei, A. Zoccolante, A. Ermini and P. Rossi-Ferrini, "Effects of Fludarabine and Gemcitabine on Human Acute Myeloid Leukemia Cell Line HL 60: Direct Comparison of Cytotoxicity and Cellular Ara-C Uptake Enhancement," Leukemia Research, Vol. 20, No. 1, 1996, pp. 37-45. doi:10.1016/0145-2126(95)00106-9

[99] H. Mueller, M. U. Kassack and M. Wiese, "Comparison of the Usefulness of the MTT, ATP and Calcein Assays to Predict the Potency of Cytotoxic Agents in Various Human Cancer Cell Lines," Biomolecular Screening, Vol. 9, No. 6, 2004, pp. 506-515. doi: $10.1177 / 1087057104265386$

[100] E. Ulukaya, F. Ozdikicioglu, A. Y. Oral and M. Dermirci, "The MTT Assay Yields a Relatively Lower Result of Growth Inhibition than the ATP Assay Depending on the Chemotherapeutic Drug Tested," Toxicol in Vitro, Vol. 22, No. 1, 2008, pp. 232-239. doi:10.1016/j.tiv.2007.08.006

[101] M. Varache-Lembège, S. Larrouture, D. Montaudon, J. Robert and A. Nuhrich, "Synthesis and Antiproliferative Activity of Aryl- and Heteroaryl-Hydrazones Derived from Xanthone Carbaldehydes," European Journal of Medicinal Chemistry, Vol. 43, No. 6, 2008, pp. 13361343. doi:10.1016/i.ejmech.2007.09.003

[102] M. D. Kars, O. D. Iseri, U. Gunduz and J. Molnar, "Reversal of Multidrug Resistance by Synthetic and Natural Compounds in Drug-Resistant MCF-7 Cell Lines," Chemotherapy, Vol. 54, No. 3, 2008, pp. 194-200. doi: $10.1159 / 000140462$

[103] H. Huang, E. Pierstorff, E. Osawa and D. Ho, "Active Nanodiamond Hydrogels for Chemotherapeutic Delivery," Nano Letters, Vol. 7, No. 11, 2007, pp. 3305-3314. doi:10.1021/n10715210

[104] M. C. Dery, C. Van Themsche, D. Provencher, A. M. Mes-Masson and E. Asselin, "Characterization of EN$1078 \mathrm{D}$, a poorly differentiated human endometrial carci- 
noma cell line: A Novel Tool to Study Endometrial Invasion In-Vitro," Reproductive Biology and Endocrinology, Vol. 5, 2007, pp. 38-39. doi:10.1186/1477-7827-5-38

[105] B. Spee, M. D. Jonkers, B. Arends, G. R. Rutteman, J. Rothuizen and L. C. Penning, "Specific Down-Regulation of XIAP with RNA Interference Enhances the Sensitivity of Canine Tumor Cell-Lines to Trail and Doxorubicin," Molecular Cancer, Vol. 5, 2006, p. 34. doi:10.1186/1476-4598-5-34

[106] N. Denora, V. Laquintana, A. Trapani, A. Lopedota, A. Latrofa, J. M. Gallo and G. Trapani, "Translocator Protein (TSPO) Ligand-Ara-C (cytarabine) Conjugates as a Strategy to Deliver Antineoplastic Drugs and to Enhance Drug Clinical Potential," Molecular Pharmaceutics, Vol. 7, No. 6, 2010, pp. 2255-2269. doi: $10.1021 / \mathrm{mp} 100235 \mathrm{w}$

[107] W. C. Shen and H. J. Ryser, "Cis-Aconityl Spacer between Daunomycin and Macromolecular Carriers: A Model of $\mathrm{pH}$-Sensitive Linkage Releasing Drug from a Lysosomotrophic Conjugates," Biochemical and Biophysical Research Communications, Vol. 102, No. 3, 1981, pp. 1048-1054. doi:10.1016/0006-291X(81)91644-2

[108] Y. Zhang, N. Wang, N. Li, T. Liu and Z. Dong, "The Antitumor Effect of Adriamycin Conjugated with Monoclonal Antibody against Gastric Cancer In-Vitro and In-Vivo," Acta Pharmaceutica Sinica, Vol. 27, No. 5, 1992, pp. 325-330.

[109] E. Aboud-Pirak, E. Hurwitz, F. Bellot, J. Schlessinger and M. Sela, "Inhibition of Human Tumor Growth in Nude Mice by a Conjugate of Doxorubicin with Monoclonal Antibodies to Epidermal Growth Factor Receptor," Proceedings of the National Academy of Sciences, Vol. 86, No. 10, 1989, pp. 3778-3781. doi:10.1073/pnas.86.10.3778

[110] C. P. Coyne, B. W. Fenwick and J. Ainsworth, "AntiNeoplastic Activity of Chemotherapeutic 'Loaded' Neutrophils against Human Mammary Carcinoma," Biotherapy, Vol. 10, No. 2, 1997, pp. 145-159. doi: $10.1007 / \mathrm{BF} 02678542$

[111] M. D. Pegram, A. Lopez, G. Konecny and D. J. Slamon, "Trastuzumab and Chemotherapeutics: Drug Interactions and Synergies," Seminars in Oncology, Vol. 27, Suppl. 11, 2000, pp. 21-25.

[112] D. Slamon and M. Pegram, "Rationale for Trastuzumab (Herceptin) in Adjuvant Breast Cancer Trials," Seminars in Oncology, Vol. 28, Suppl. 3, 2001, pp. 13-19. doi:10.1053/sonc.2001.22812

[113] E. P. Winer and H. J. Burstein, "New Combinations with Herceptin in Metastatic Breast Cancer," Oncology, Vol. 61, Suppl. 2, 2001, pp. 50-57. doi:10.1159/000055402
[114] S. Kim, C. N. Prichard, M. N. Younes, Y. D. Yazici, S. A. Jasser, B. N. Bekele and J. N. Myers, "Cetuximab and Irinotecan Interact Synergistically to Inhibit the Growth of Orthotopic Anaplastic Thyroid Carcinoma Xenografts in Nude Mice," Clinical Cancer Research, Vol. 12, No. 2, 2006, pp. 600-607. doi:10.1158/1078-0432.CCR-05-1325

[115] M. Landriscina, F. Maddalena, A. Fabiano, A. Piscazzi, O. La Macchia and M. Cignarelli, "Erlotinib Enhances the Proapoptotic Activity of Cytotoxic Agents and Synergizes with Paclitaxel in Poorly-Differentiated Thyroid Carcinoma Cells," Anticancer Research, Vol. 30, No. 2, 2010, pp. 473-480.

[116] F. Ciardiello, R. Bianco, V. Damiano, S. De Lorenzo, S. Pepe, S. De Placido, Z. Fan, J. Mendelsohn, A. Bianco and G. Tortora, "Antitumor Activity of Sequential Treatment with Topotecan and Anti-Epidermal Growth Factor Receptor Monoclonal Antibody C225," Clinical Cancer Research, Vol. 5, No. 4, 1999, pp. 909-916.

[117] K. D. Lynn, D. G. Udugamasooriya, C. L. Roland, D. H. Castrillon, T. J. Kodadek and R. A. Brekken, "GU81, a VEGFR2 Antagonist Peptoid, Enhances the Anti-Tumor Activity of Doxorubicin in the Murine MMTV-PyMT Transgenic Model of Breast Cancer," BMC Cancer, Vol. 10, 2010, p. 397. doi:10.1186/1471-2407-10-397

[118] L. Zhang, D. Yu, D. J. Hicklin, J. A. Hannay, L. M. Ellis and R. E. Pollock, "Combined Anti-Fetal Liver Kinase 1 Monoclonal Antibody (Anti-VEGFR) and Continuous Low-Dose Doxorubicin Inhibits Angiogenesis and Growth of Human Soft Tissue Sarcoma Xenografts by Induction of Endothelial Cell Apoptosis," Cancer Research, Vol. 62, No. 7, 2002, pp. 2034-2042.

[119] L. B. Shih, D. M. Goldenberg, H. Xuan, H. W. Lu, M. J. Mattes and T. C. Hall, "Internalization of an Intact Doxorubicin Immunoconjugate," Cancer Immunology, Immunotherapy, Vol. 38, No. 2, 1994, pp. 92-98. doi:10.1007/BF01526203

[120] H. J. Hansen, G. L. Ong and H. Diril, "Internalization and Catabolism of Radiolabeled Antibodies to the MHC ClassII Invariant Chain by B-Cell Lymphomas," Biochemical Journal, Vol. 320, 1996, pp. 293-300.

[121] A. C. Stan, D. L. Radu, S. Casares, C. A. Bona and T. D. Brumeanu, "Antineoplastic Efficacy of Doxorubicin Enzymatically Assembled on Galactose Residues of a Monoclonal Antibody Specific for the Carcinoembryonic Antigen," Cancer Research, Vol. 59, No. 1, 1999, pp. 115121.

[122] M. Pimm, V, M. A. Paul, T. Ogumuyiwa and R. W. Baldwin, "Biodistribution and Tumour Localization of a Daunomycin-Monoclonal Antibody Conjugate in Nude Mice and Human Tumour Xenografts," Cancer Immunology, Immunotherapy, Vol. 27, No. 3, 1988, pp. $267-$ 271. 